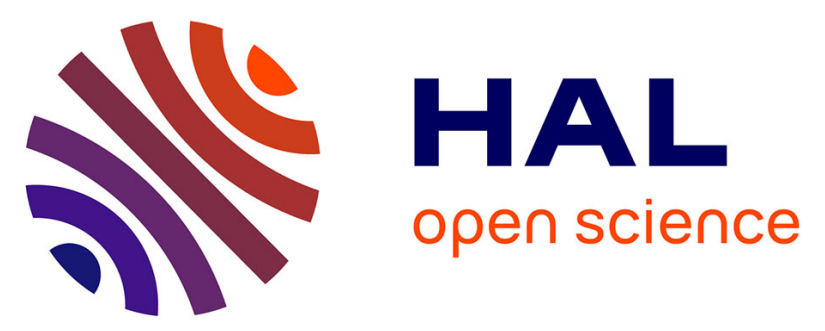

\title{
Effect of Eshelby twist on core structure of screw dislocations in molybdenum: Atomic structure and electron microscope image simulations
}

Roman Gröger, Karleen J Dudeck, Peter D Nellist, Vaclav Vitek, Peter B Hirsch, D J H Cockayne

\section{To cite this version:}

Roman Gröger, Karleen J Dudeck, Peter D Nellist, Vaclav Vitek, Peter B Hirsch, et al.. Effect of Eshelby twist on core structure of screw dislocations in molybdenum: Atomic structure and electron microscope image simulations. Philosophical Magazine, 2011, pp.1. 10.1080/14786435.2011.562474. hal-00681619

\section{HAL Id: hal-00681619 https://hal.science/hal-00681619}

Submitted on 22 Mar 2012

HAL is a multi-disciplinary open access archive for the deposit and dissemination of scientific research documents, whether they are published or not. The documents may come from teaching and research institutions in France or abroad, or from public or private research centers.
L'archive ouverte pluridisciplinaire $\mathbf{H A L}$, est destinée au dépôt et à la diffusion de documents scientifiques de niveau recherche, publiés ou non, émanant des établissements d'enseignement et de recherche français ou étrangers, des laboratoires publics ou privés. 


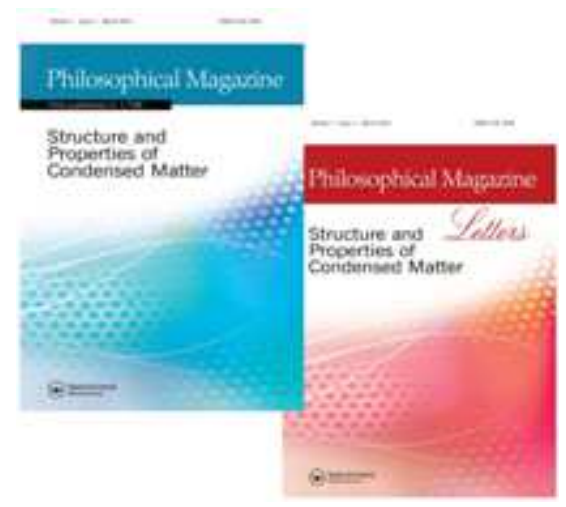

\section{Effect of Eshelby twist on core structure of screw dislocations in molybdenum: Atomic structure and electron microscope image simulations}

\begin{tabular}{|c|c|}
\hline Journal: & Philosophical Magazine \& Philosophical Magazine Letters \\
\hline Manuscript ID: & TPHM-10-Nov-0490.R2 \\
\hline Journal Selection: & Philosophical Magazine \\
\hline $\begin{array}{r}\text { Date Submitted by the } \\
\text { Author: }\end{array}$ & 09-Feb-2011 \\
\hline Complete List of Authors: & $\begin{array}{l}\text { Gröger, Roman; Academy of Sciences of the Czech Republic, } \\
\text { Institute of Physics of Materials } \\
\text { Dudeck, Karleen; University of Oxford, Department of Materials } \\
\text { Nellist, Peter; University of Oxford, Department of Materials } \\
\text { Vitek, Vaclav; University of Pennsylvania, Department of Materials } \\
\text { Science and Engineering } \\
\text { Hirsch, Peter; University of Oxford, Department of Materials } \\
\text { Cockayne, D; University of Oxford, Department of Materials }\end{array}$ \\
\hline Keywords: & dislocation structures, free surface, HREM \\
\hline Keywords (user supplied): & screw dislocation, thin foil, Eshelby twist \\
\hline
\end{tabular}

\section{SCHOLARONE Manuscripts}




\title{
Effect of Eshelby twist on core structure of screw dislocations in molybdenum: Atomic structure and electron microscope image simulations
}

\author{
R. Gröger ${ }^{1,}$, K. J. Dudeck ${ }^{2,3}$, P. D. Nellist ${ }^{2}$, V. Vitek ${ }^{4}$, P. B. Hirsch ${ }^{2}$, D.J.H. Cockayne ${ }^{2}$ \\ ${ }^{1}$ Institute of Physics of Materials, Academy of Sciences of the Czech Republic, Žižkova 22, 61662 \\ Brno, Czech Republic \\ ${ }^{2}$ Department of Materials, University of Oxford, Parks Road, Oxford OX1 3PH, UK \\ ${ }^{3}$ Department of Materials Science and Engineering, McMaster University, Hamilton L8S 4L7, Canada \\ ${ }^{4}$ Department of Materials Science and Engineering, University of Pennsylvania, 3231 Walnut Street, \\ Philadelphia, PA 19104, USA
}

\begin{abstract}
This paper addresses the question as to whether the core structure of screw dislocations in Mo in the bulk can be obtained from HREM images of such dislocations viewed end-on in a thin foil. Atomistic simulations of the core structure of screw dislocations in elastically anisotropic Mo are carried out using Bond Order Potentials. These simulations take account automatically of the effects of the surface relaxation displacements (anisotropic Eshelby twist). They show that the differential displacements of the atoms at the surface are different with components perpendicular to the Burgers vector about five times larger than those in the middle of the foil, the latter being characteristic of the bulk. Nye tensor plots show that the surface relaxation stresses strongly affect the incompatible distortions. HREM simulations of the computed structure reflect the displacements at the exit surface, modified by interband scattering and the microscope transfer function. Nye tensor plots obtained from the HREM images show that interband scattering also affects the incompatible distortions. It is concluded that it would be very difficult to obtain information on the core structure of screw dislocations in the bulk Mo from HREM images, even under ideal experimental conditions, and that quantitative comparisons between experimental and simulated images from assumed model structures would be essential.
\end{abstract}

\section{Keywords:}

screw dislocation, thin foil, Eshelby twist, free surface, HREM

* Corresponding author. E-mail: groger@ipm.cz 


\section{Introduction}

It is generally accepted that the plastic deformation of pure BCC metals is controlled by $1 / 2\langle 111\rangle$ screw dislocations (for reviews see [1-6]). The reason is, as first suggested by Hirsch [7], that these dislocations possess non-planar cores extending into several planes of the $\langle 111\rangle$ zone. This core nonplanarity leads to a very high Peierls stress that is responsible for a rapid increase of the flow stress with decreasing temperature and increasing strain rate. The non-planar core also produces a strong dependence of the flow stress on the orientation of the crystal relative to the loading axes and related break-down of the Schmid law. Such non-planar spreading of the core has, indeed, been found in all atomistic studies performed in the last forty years (for reviews see [2, 6, 8-11]). Transmission electron microscopy (TEM) observations of dislocation substructures after deformation [12-15] and in-situ studies [16-21] of the glide of dislocations reveal the presence of long screw dislocation segments that indicates their very low mobility relative to the dislocations of other orientations. However, no direct experimental observation confirms the non-planar core structure unambiguously.

In recent years, advances in high resolution electron microscopy (HREM) spawned an interest in utilising this technique to investigate in detail the structure of dislocation cores in thin metallic foils of BCC metals. Two attempts had been made to observe the core structure of the $1 / 2\langle 111\rangle$ screw dislocation in molybdenum by HREM $[22,23]$. Apart from severe experimental difficulties, these studies are burdened by two fundamental problems. Firstly, the image principally reflects the displacements at the exit surface, and because of interband scattering the image peaks are displaced relative to the actual positions of atoms at the surface [24]. Secondly, the screw dislocation normal to and terminating at traction-free surfaces generates the so-called Eshelby twist. This deformation field, derived theoretically for an isotropic material by Eshelby and Stroh [25], is characterised by counterrotations of the upper and lower parts of the foil and it is much larger than the core displacements in the bulk. In an attempt to separate the core and Eshelby twist displacements, Mendis et al. [23] revisited the concept of the Nye tensor and showed that it captures only the incompatible part of the deformation field. Their Nye tensor plots show that the displacements due to both the Eshelby twist and the dynamical scattering of the electrons behave as compatible deformations and that, therefore, they do not appear in the components of the Nye tensor.

Since the HREM image reflects the displacements at the exit surface, the fundamental questions are: (i) whether the HREM observations relate to the core displacements in the bulk, and (ii) whether the stresses associated with the Eshelby twist produce such significant changes in the dislocation core structure that the relation to the core structure in the bulk becomes obscured. The objectives of this paper are to elucidate these points by carrying out an atomistic simulation of the $1 / 2\langle 111\rangle$ screw dislocation in a thin foil of Mo of thickness typical for HREM observations, and to simulate the HREM images of the calculated structure. The atomic displacements of the relaxed structure automatically contain the surface-induced relaxation displacements, and the effects of the surface relaxation stresses on the core structure. These displacements can then be identified as the difference between the relaxed positions of atoms in the foil and their relaxed positions in a block that is periodic in the direction parallel to the dislocation line, i. e. without free surfaces. Finally, the calculated relaxed atomic positions in the foil can be used as input for the image analysis. This provides the positions of image 
peaks at the exit surface, which reflect the Eshelby twist and core displacements, plus the displacements due to the interband scattering and the microscope transfer function.

\section{Atomistic evaluation of the Eshelby twist and its separation from the core displacements}

\subsection{Computational model}

The object of the calculations is to determine the atomic positions around a $1 / 2[111]$ screw dislocation that is normal to a thin foil of Mo, and compare them with those in an infinite crystal. The procedure adopted in this paper requires two atomistic simulations in which the energy of a cylindrical block (with its axis of revolution coincident with the screw dislocation) is minimised with respect of the positions of the atoms. To obtain the atomistic positions near the core in an infinite crystal we utilise an atomic block which is effectively of infinite thickness. To ensure that the atoms in the block assume positions that approximate to those in a crystal of infinite radius, we impose the boundary condition that the atoms in an outer rim of the block assume the positions given by the anisotropic linear-elastic strain field of the screw dislocation in an infinite lattice. After inserting the dislocation into the block, the two terminating (111) planes contain ledges that arise necessarily due to the screw character of the dislocation. We do not give the atoms at these surfaces any special treatment and let them relax as it is done with any other atom in the block.

To determine the positions of the atoms in a thin foil we utilise a cylindrical block of the same radius but of finite thickness, terminated by traction-free surfaces normal to its axis of revolution. To ensure the correct boundary conditions in the radial direction appropriate to those expected for a foil of infinite radius we need the linear-elastic solution of the Eshelby twist strain field for an anisotropic lattice. Such a solution is not known. Instead, as an approximation, we impose a boundary condition consisting of the anisotropic linear-elastic strain field for the screw dislocation in an infinite crystal, plus the Eshelby-Stroh solution [25] of the additional tangential displacements due to the Eshelby twist in an isotropic crystal. In that way the constraint of the part of the foil outside the simulated block of finite radius is taken into account in an approximate manner. In the former, the elastic moduli of molybdenum are taken from Ref. [26]: $C_{11}=464.7 \mathrm{GPa}, C_{12}=161.5 \mathrm{GPa}$ and $C_{44}=108.9 \mathrm{GPa}$. Since the anisotropy ratio $2 C_{44} /\left(C_{11}-C_{12}\right)$ is 0.7 , molybdenum is not strongly elastically anisotropic. Moreover, the Eshelby-Stroh formula for tangential displacements is independent of elastic moduli, which avoids the ambiguity of reducing the set of three anisotropic elastic moduli above to their two isotropic counterparts. Both these observations further justify the use of the isotropic Eshelby-Stroh displacement field far away from the dislocation. The effect of the Eshelby twist is then determined by the differences of the displacements computed in the thin foil and infinite crystal.

The reason for making the blocks cylindrical is to avoid complications associated with possible unequal distances from the dislocation centre to the lateral face of the block that arise for other geometries. This is particularly important in this case because the displacements due to the Eshelby twist are long range. In both simulations, the coordinate system was chosen such that the axis of revolution of the cylinders coincides with the $z$ axis that is parallel to the [111] direction and thus to both the dislocation line and the Burgers vector, the $x$ axis parallel to the [ $\overline{1} 2 \overline{1}]$ direction and the $y$ axis 
parallel to the [101] direction. This choice of axes makes the coordinate system right-handed. In both relaxation calculations below we used the Bond Order Potential (BOP) for Mo [27] that captures the mixed nearly free electron and covalent character of bonding in this metal.

In calculations for the infinite crystal we utilise an atomic block that is periodic along the dislocation line and effectively infinite in the plane perpendicular to the dislocation line. The repeat cell is composed of three (111) planes that represent one 1/2[111] period of the BCC lattice with the outer radius equal to $15 a$, where $a=3.1472 \AA$ is taken as the lattice parameter of Mo. The $1 / 2[111]$ screw dislocation was inserted in the middle of the block in the direction parallel to the $z$ axis by displacing all atoms according to the anisotropic linear-elastic strain field of the dislocation in an infinite lattice. The total number of atoms in this block was 1221. During the relaxation, 675 atoms at the distance from the core $10<r / a<15$, were held fixed, while the positions of 546 atoms at the distances $r / a \leq 10$ were adjusted. The former group of atoms thus represents an outer rim of the block in which the positions of atoms are determined by the linear-elastic anisotropic solution for an infinite Volterra dislocation. The positions of the atoms that constitute the inner part of the block are found by the relaxation during which all these atoms are allowed to move in all directions. Close to the centre of the dislocation these atomic positions define the core structure. The relaxation was terminated when the magnitudes of forces acting at all atoms fell below $0.005 \mathrm{eV} / \AA$. The relaxed position of an atom $i$ can then be written as

$$
\mathbf{x}_{\infty}^{i}=\mathbf{X}^{i}+\mathbf{u}_{\text {dislo }}^{i},
$$

where $\mathbf{X}^{i}$ is the initial position of the atom $i$ in the perfect lattice, and $\mathbf{u}_{\text {dislo }}^{i}$ the displacement of this atom due to the dislocation. The positions $\mathbf{x}_{\infty}^{i}$ are the atomic positions in a block that is infinite in all directions and contains a screw dislocation in the middle.

In the second simulation, for the thin foil with the $1 / 2[111]$ screw dislocation perpendicular to the traction-free foil surfaces, the simulation cell is constructed by taking the relaxed periodic block of 1221 atoms from the first simulation and copying it 18 times along the $z$ direction. This cell, which represents the foil, had the following additional properties. The origin of the $z$ axis was chosen such that $z= \pm h / 2$ correspond to the positions of the two free surfaces terminating the block in the $z$ direction. Along the $z$ direction the block was composed of 19 complete periods, i.e. 57 atomic planes of the (111) type with the layer-to-layer distance of $a / 2 \sqrt{3}$. Hence, the total thickness of the simulated foil was $5.09 \mathrm{~nm}$. The diameter of the block in the $(x, y)$ plane was about 30 lattice periods and the block contained 23199 atoms of which 12825 atoms were located in the outer rim (i. e. at $10<r / a<15)$ and the remaining 10374 atoms with positions $r / a \leq 10$ in the inner part of the block. We imposed the traction-free conditions on the upper and lower flat surfaces by displacing all atoms in this block by the isotropic Eshelby-Stroh displacement field [25]

$$
u_{\theta}(r, z)=-\frac{b}{2 \pi} \sum_{n=0}^{\infty}(-1)^{n}\left[\frac{r}{w_{n-}+\sqrt{w_{n-}^{2}+r^{2}}}-\frac{r}{w_{n+}+\sqrt{w_{n+}^{2}+r^{2}}}\right] .
$$

Here, $b$ is the magnitude of the Burgers vector, $r$ the distance from the dislocation, and 
$w_{n \pm}=(2 n+1) h / 2 \pm z$. Only the terms of the series corresponding to $n \in\langle 0,10\rangle$ are considered; all higher order terms not accounted for in the sum have negligible effect on the displacement field. During the subsequent minimisation of the energy, only the atoms in the inner part of the block move while those in the outer rim are held fixed. Consequently, the displacements of the atoms in the outer rim are given by the superposition of the elastic long-range displacement field of a straight dislocation in an anisotropic crystal and the isotropic Eshelby-Stroh field (2). This block has many more degrees of freedom than the infinite periodic block and thus the relaxation approached the stable equilibrium much more slowly than in the periodic case. Hence we stopped the relaxation of the atomic positions in this block when the forces on all atoms fell below $0.06 \mathrm{eV} / \AA$. Further relaxation is unlikely to affect the positions of atoms near the centre of the dislocation. The calculated position $\mathbf{x}_{f}^{i}$ of each atom $i$ in the inner part of the block is then

$$
\mathbf{x}_{f}^{i}=\mathbf{x}_{\infty}^{i}+\mathbf{u}_{t w i s t}^{i}=\mathbf{X}^{i}+\mathbf{u}_{\text {dislo }}^{i}+\mathbf{u}_{\text {twist }}^{i},
$$

where $\mathbf{x}_{\infty}^{i}$ is the position of the atom obtained from the first simulation, and $\mathbf{u}_{\text {twist }}^{i}$ the displacement due to the Eshelby twist (including the effects of surface relaxation on the core displacements) owing to the traction-free surfaces on the upper and lower flat surfaces of the cylinder. From symmetry, the Eshelby twist vanishes in the middle plane of the foil (i.e. at $z=0$ ) and, therefore, the atoms in this plane are displaced in the same way as in the periodic block. This provides a check on the calculation. The field $\left\{\mathbf{x}_{f}^{i}, i=1 . . N\right\}$, where $N$ is the number of atoms, then represents the positions of atoms in a thin foil that is finite in the $z$ direction but (approximately) infinite in the $(x, y)$ plane. These positions are used in the image simulation in Section 3.

\subsection{Structure of the dislocation core}

In Figs. 1 and 2 we show the maps of the differential displacements parallel and perpendicular to the dislocation line (and to the Burgers vector), respectively, for three slices taken from the upper and lower surfaces and from the centre of the relaxed block that represents the foil. The atomic arrangement is shown in the projection perpendicular to the direction of the dislocation line. The circles stand for the atoms in the three consecutive (111) atomic planes (differentiated by shading) and the lengths of the arrows are proportional to the magnitudes of the relative displacements of neighbouring atoms either in the direction parallel (screw displacements; Fig. 1) or perpendicular (further called edge displacements; Fig. 2) to the dislocation line, i.e. corresponding to the relative displacements in the $z$ direction and in the $(x, y)$ plane, respectively. The colours of atoms are chosen such that they get darker with increasing position along the $z$ direction in the ideal lattice. For example, when plotting Figs. 1a and $2 \mathrm{a}$ (upper surface), black corresponds to the atoms in the (111) plane that terminates the foil at the upper surface, grey are the atoms in the next (111) plane below, and white correspond to the atoms in the third (111) plane from the upper surface. Figs. 1a and 2a correspond to the slice composed of three (111) planes at the upper surface and Figs. 1c and 2c to the slice composed of three (111) planes at the lower surface. Figs. $1 b$ and $2 b$ show the central slice. The magnitudes of the edge displacements are much smaller than the magnitudes of the screw displacements. However, the edge displacements near 
the surfaces are about 5 times larger than those in the centre of the foil and, therefore, the displacements in Fig. $2 b$ are scaled by a factor of 10 while those in Figs. $2 a, c$ only by a factor of 2 . The atoms in the centre slice, shown in Figs. $1 b$ and $2 b$, experience very similar displacements as in the case of an infinite straight screw dislocation and thus the displacement maps shown in Figs. $1 \mathrm{~b}$ and $2 \mathrm{~b}$ are practically the same as those presented in Refs. [27, 28]. In the centre of the foil the core possesses very closely both the [111] threefold screw axis and [101] diad symmetry, i. e. it is practically the same as the non-degenerate core found in studies of infinitely long straight screw dislocations [27, 28]. However, near the surfaces the [ $\overline{1} 01]$ diad symmetry is removed. This is similar to the situation encountered in the case of infinite dislocations when an external shear stress in the (101) plane or tension along an axis within the standard triangle is applied [28-30]. The core extends into the ( $\overline{1} 01)$ plane on both surfaces, while the displacements in the $(\overline{1} 10)$ and $(0 \overline{1} 1)$ planes are suppressed. Moreover, as mentioned above, the edge displacements in the surface slices are much larger than those in the middle of the foil. This means that there are significant atomic relaxations in the direction perpendicular to the Burgers vector on both the upper and lower surfaces.

Further insight into the effect of the free surface on the structure of the dislocation core can be gained by considering the directions of edge displacements. It was shown in [30] that in the case of infinite straight dislocations these displacements are practically parallel to the lines connecting the neighbouring atoms so that the bond angles remain close to those found in the ideal BCC structure. This means that the edge displacements produce a predominantly local expansion. The same is found in the middle of the foil (Fig. 2b) where the displacements due to the Eshelby twist are negligible. On the other hand, near the surfaces (Figs. 2a,c) the Eshelby twist rotates the atomic bonds towards the directions perpendicular to those in the twist-free region (Fig. 2b). Hence, near the surfaces the system minimises its energy by keeping the local density as unchanged as possible and thus the nearest neighbour separations constant, whilst the bond angles are forced to change.

\subsection{Determination of the anisotropic Eshelby twist}

The anisotropic Eshelby twist can now be calculated by comparing the relaxed positions of atoms in the foil with those in the cell that is periodic in the $z$ direction and effectively infinite in the $(x, y)$ plane, both of which were obtained in Subsection 2.1. The difference

$$
\mathbf{u}_{t w i s t}^{i}=\mathbf{x}_{f}^{i}-\mathbf{x}_{\infty}^{i}
$$

where $\mathbf{x}_{\infty}^{i}$ and $\mathbf{x}_{f}^{i}$ are obtained from Eqs. (1) and (3), respectively, then measures the changes in the atomic positions induced by the traction-free boundaries of the foil. Since the interatomic potential used was fitted to reproduce the anisotropic elastic moduli of Mo, the displacements $\mathbf{u}_{\text {twist }}^{i}$ represent the Eshelby twist evaluated fully anisotropically with the constraint that these displacements approach the isotropic Eshelby-Stroh solution (2) at $r / a=10$. For an isotropic material it was shown in [25] that the Eshelby twist causes opposite tangential displacements on the two free surfaces and thus necessarily counter-rotations of the upper and lower parts of the block. Similar response is expected in anisotropic crystals. This is demonstrated in Fig. 3 where we show the $(x, y)$ component of the 
displacement field of the anisotropic Eshelby twist, $\mathbf{u}_{\text {twist }}^{i}$, determined according to Eq. (4) (black arrows) and those of the isotropic Eshelby-Stroh solution (red arrows). The displacements, i.e. the lengths of arrows in this figure, are scaled by a factor of 5. The dots represent positions of atoms in three consecutive (111) planes (not differentiated) in the perfect crystal at the upper surface (Fig. 3a) and at the lower surface (Fig. 3b), respectively; the triangles mark the centre of the dislocation in the infinite periodic block. The differences in the displacements of the atoms nearest to the core in the upper and lower surfaces, respectively, result because the depicted atoms have different distances from the middle plane of the foil. One can clearly see that the displacement field $\mathbf{u}_{t w i s t}^{i}$ has a predominantly tangential character and represents rotations in opposite directions in the upper and lower parts of the foil. Since these tangential displacements vary along the $z$ direction and become negligible for $z=0$, the induced twist is not uniform throughout the block but varies with both the depth in the foil, i. e. coordinate $z$, and the distance from the centre of the dislocation. It is apparent from this figure that the main differences between $\mathbf{u}_{\text {twist }}^{i}$ component in the $(x, y)$ plane and the Eshelby-Stroh linear-elastic displacements (Eq. 2) occur close to the core. This is seen more clearly in Fig. 4 which shows these two displacements for the three (111) planes at the lower surface (Fig. 3b) as a function of $r / a$; the main deviations occur for $r / a<3$. At larger values of $r / a$ the varying deviations of the values of $\mathbf{u}_{t w i s t}^{i}$ at a given $r / a$ relate to the effect of anisotropy. We note that the displacements calculated by the atomistic simulation approach those of the isotropic Eshelby-Stroh displacements with increasing $r / a$. To check the sensitivity of the displacements near the core to the boundary conditions at $r / a=10$, a calculation was performed with stress-free boundary conditions at $r / a=10$. The differences were less than $10 \%$ at $r / a<3$.

We are now in a position to answer the first question stated in the Introduction, i. e. whether or not the Eshelby twist affects the dislocation core. It will be convenient to utilize the concept of the Nye tensor [31] within which an arbitrary displacement field can be represented by a continuous field of infinitesimal (i. e. virtual) dislocations. The density of the Burgers vectors of these infinitesimal dislocations is then represented by a symmetric tensor $\boldsymbol{\alpha}$. It satisfies the Bianchi identity $\nabla \cdot \boldsymbol{\alpha}=\mathbf{0}$ which constrains the dislocation network such that no dislocation begins or ends inside the body [32]. The component $\alpha_{i j}$ represents the density of the infinitesimal dislocations whose line directions are parallel to $x_{i}$ and their Burgers vectors are parallel to $x_{j}$. An important property of the Nye tensor is that it describes only the incompatible part of the elastic-plastic deformation field [23, 32]. Therefore, any compatible distortion of the lattice for which the displacement field remains single-valued and the strain field integrable does not affect the Nye tensor. Consequently, if the Eshelby twist did not affect the dislocation core, the Nye tensor plots calculated for the two surface slices would have to be identical to that taken from the middle of the foil. In Fig. 5 we show the three relevant components of the Nye tensor for the upper and lower surfaces and the middle of the foil. Since the dislocation line is parallel to the $z$ direction, $\alpha_{33}$ corresponds to the screw component of the dislocation density. Similarly, $\alpha_{31}$ and $\alpha_{32}$ relate to the two edge components, for the Burgers vectors parallel to $x$ and $y$, respectively. We represent the dislocation core by a region around the dislocation in which the 
displacements of atoms relative to their positions in the ideal lattice, and thus also $\alpha_{i j}$, are larger than some threshold. Since the Eshelby twist strongly affects the edge component of this field (as seen in Fig. 5), i.e. the relative displacements of atoms perpendicular to the slip direction, the dislocation core close to the two free surfaces is very different from that in the center of the foil. We thus conclude that the Eshelby twist strongly affects the dislocation core near the foil surfaces but not in the bulk of the foil. The Eshelby twist thus induces both compatible deformation away from the dislocation core and the incompatible deformation in the core region. The latter is not surprising since the surface relaxation strains are very large. For example, using the Eshelby-Stroh formula (Eq. 2) [21], the shear strains at about $3 \AA$ from the core are about 0.1 . The existence of these large surface strains is further corroborated by the edge components of the differential displacement maps shown in Fig. 2, where the surface strains (Figs. 2a,c) are a factor of 5 larger than the strains in the middle of the foil (Fig. 2b).

\section{HREM Image analysis}

Simulated HREM images were calculated using the multislice method incorporated into the QSTEM software package [33, 34]. A model consisting of atoms of Mo placed at the set of atomic coordinates given by $\mathbf{x}_{f}^{i}$ was used, with the illuminating beam taken to be incident parallel to the [111] direction of the sample (i.e. parallel to the dislocation). Reference images were also calculated for a perfect reference crystal with a set of atomic coordinates given by $\mathbf{X}^{i}$. In both cases the imaging conditions were: an accelerating voltage of $300 \mathrm{kV}$, an incident beam divergence of $0.2 \mathrm{mrad}$ and a Gaussian distributed defocus spread (arising from chromatic aberration) with a standard deviation of $2.9 \mathrm{~nm}$. No lens aberrations were included in the calculation. Although these zero-aberration conditions do not give weak-phase contrast, the sample here is thick enough to be strongly dynamical leading to significant amplitude changes in the exit wave. Furthermore, the presence of aberrations would lead to increased delocalisation in the image and greater complexity in the contrast from atomic columns. The simulated image of the perfect crystal (Fig. 6a) shows that the zero-aberration conditions assumed here gave simple peaks with a single maximum at the location of each atomic column. Introducing aberrations was found to result in a more complex "donut" contrast for each atomic column. With spherical aberration correctors becoming more commonplace, the zero-aberration condition is now feasible to achieve experimentally. The simulated image of the relaxed model including the Eshelby twist (coordinate set $\mathbf{x}_{f}^{i}$ ) under these imaging conditions is shown in Fig $6 \mathrm{~b}$. The effect of the Eshelby twist on the image can be clearly seen where the bright peaks are now displaced and asymmetric bright-dark contrast is seen. Closer to the core, the dark regions are not as apparent.

In order to determine the extent to which the images can be compared with the atomistic model, the intensity peak for each atomic column was located, and the displacement from the perfect crystal position computed. The images were simulated with a pixel size of $0.03 \AA$, and this represents the precision to which the peaks were located. The precision to which peaks in an HRTEM image can be located is a function of image resolution and signal-to-noise ratio, but the precision used in the image 
simulations here is similar to the highest precisions that have been observed experimentally [35].

\section{Results and implications}

This paper raises the question of what information on the core structure of screw dislocations can be obtained from HREM images of such dislocations viewed end-on. There are two issues: (i) the extent to which the images reflect accurately the surface core structure of the dislocation, and (ii) the information that can be gained on the core structure in the bulk.

On the first point, the images reflect the displacements at the surface, but modified by interband scattering [24] and the microscope transfer function. Fig. 7 shows a comparison of the absolute displacement (scaled 5 times) at the exit surface around the screw dislocation obtained from atomistic simulations (black) and from the peak positions in the image analysis (red). Close to the core the directions and magnitudes of the displacements obtained in these two ways differ significantly, while further away the directions are similar. The displacements from the image peak positions are slightly larger than those from the atomistic simulations. The discrepancies between the two plots show the effect of interband scattering and the microscope transfer function on the image peak positions, which are largest close to the dislocation core.

Fig. 8 compares the edge component of the differential displacement maps obtained from atomistic simulations (a) and from image analysis (b). The arrows in both plots have been scaled by a factor of 2. While on the whole the magnitudes of the differential displacements are comparable, there are significant differences in detail, again illustrating the effects of interband scattering and the microscope transfer function.

Mendis et al. [23] simulated images of a singular screw dislocation in an elastically isotropic thin foil including the Eshelby twist using the Eshelby-Stroh formula [24]. They found that the Nye plots were featureless, showing that the effects of interband scattering and the microscope transfer function did not introduce apparent incompatible deformation. Fig. 9 shows a comparison of Nye plots of the edge components $\alpha_{31}$ and $\alpha_{32}$ obtained from our atomistic calculations (Fig. 9a) and from the positions of image peaks (Fig. 9b). While some features are similar, there are clearly some significant differences. We conclude that in these image simulations both the interband scattering and the microscope transfer function do affect the incompatible as well as the compatible deformations. However the magnitudes of $\alpha_{i j}$ in Fig. $9 \mathrm{~b}$ reflect those at the exit surface, which are considerably greater than those expected from the atomistic simulations in the bulk. The assumption made in a previous image simulation of such dislocations by Mendis et al. [23], that the interaction between surface relaxation stresses and the core structure is negligible, appears to be invalid. The possibility of such an interaction was raised in [36], but its magnitude was not known.

It should be emphasised that the interband scattering is sensitive to image conditions; e.g. small changes in thickness or orientation can result in considerable changes in interband scattering. The displacements observed in the experimental images of Sigle [22] and Mendis et al [23], which do not conform to those expected from Fig. 5, may well have been affected by small changes of crystal 
orientation and possibly thickness. The simulations of Mendis et al. [23] were carried under different imaging conditions from those used in the present paper. Repeating our analysis, but using the imaging conditions of Mendis et al. (data not shown), gives displacements about 3 times smaller than those plotted in Fig. 7, and Nye tensor plots with values two orders of magnitude smaller than those in Fig. 9. Changing the microscope transfer function can clearly have a very significant effect on the measurement of the incompatible deformation using HREM.

In conclusion, the atomistic simulation of the core structure of the screw dislocation normal to the surface of a thin foil of Mo has shown that the surface relaxation displacements are large and that the surface relaxation stresses have a large effect on the core structure at the surface, with serious implications on the interpretation of HREM images of such dislocations viewed end-on. The results of the image simulations in this paper suggest that the displacements, differential displacements and Nye tensor plots obtained from the image peak positions do not reflect accurately the structure at the surface. On the other hand, the apparent displacements derived from the peak positions in the image, which include the effects of interband scattering and the microscope transfer function, are large enough to be measured in an HREM experiment. To that extent, therefore, the much larger displacements at the surface expected from the atomistic simulations are helpful, and suitable for comparison with image simulation from assumed models. But to obtain any meaningful information of the core displacements in the bulk, quantitative comparison between experimental images and those obtained from assumed structures would be essential. Furthermore, the magnitude of edge component of the core displacement in the bulk $(\sim 0.01 \mathrm{~nm})$ is smaller than the currently demonstrated precision to which peak positions have been measured in a typical HREM experiment. A similar order of magnitude of these edge displacements is obtained also with other boundary conditions, e.g. when all faces of the cylinder are traction-free. Data that would provide sufficient sensitivity for the core displacements to be determined will require improvements in image signal-to-noise ratio, and therefore microscope and sample stability and cleanliness.

\section{Acknowledgments}

We thank Yuri Mishin for sharing his code for plotting the Nye tensor maps. RG acknowledges support from the Czech Science Foundation (Grant No. P204/10/0255) and from the Academy of Sciences of the Czech Republic (Research Project No. AV0Z20410507). VV acknowledges support from the US Department of Energy, BES, Grant No. DE-PG02-98ER45702. PDN and PBH gratefully acknowledge the provision of facilities by the Department of Materials, University of Oxford. The access to the METACentrum supercomputing facilities provided under the research intent MSM6383917201 is highly appreciated. 


\section{References}

[1] J.W. Christian, Metall. Trans. A 14 (1983) p. 1237.

[2] M.S. Duesbery, in Dislocations in Solids, edited by F. R. N. Nabarro, vol. 8, (Elsevier, Amsterdam, 1989) p. 67.

[3] S. Takeuchi, Radiat.. Eff. Defect Solid 148 (1999) p. 333.

[4] M.S. Duesbery, V. Vitek and J. Cserti, in Understanding Materials: A Festschrift for Sir Peter Hirsch, edited by C. J. Humphreys, vol. (The Institute of Materials, London, 2002) p. 165.

[5] A. Seeger, Z. Metallk. 93 (2002) p. 760.

[6] V. Vitek and V. Paidar, in Dislocations in Solids, edited by J. P. Hirth, vol. 14, (Elsevier, Amsterdam, 2008) p. 439.

[7] P.B. Hirsch, in Proc. 5th Int. Conf. Crystallography, edited by vol. Cambridge University, oral communication, 1960) p. 139.

[8] V. Vitek, Cryst. Latt. Def. 5 (1974) p. 1.

[9] M.S. Duesbery and G.Y. Richardson, CRC Cr. Rev. Sol. State 17 (1991) p. 1.

[10] V. Vitek, Prog. Mater. Sci. 36 (1992) p. 1.

[11] M.S. Duesbery and V. Vitek, Acta Mater. 46 (1998) p. 1481.

[12] T.E. Mitchell, R.A. Foxall and P.B. Hirsch, Philos. Mag. A 8 (1963) p. 1895.

[13] M.S. Duesbery, R.A. Foxall and P.B. Hirsch, J. Phys. Colloques 27 (1966) p. 193.

[14] R.A. Foxall, M.S. Duesbery and P.B. Hirsch, Can. J. of Phys. 45 (1967) p. 607.

[15] M.J. Mills, N.L. Baluc and P.M. Sarosi, Microsc. Res. Techniq. 69 (2006) p. 317.

[16] D. Vesely, Phys. Stat. Sol. 29 (1968) p. 675.

[17] F. Louchet and L. Kubin, Acta Metall. 23 (1975) p. 17.

[18] H. Matsui and H. Kimura, Mat. Sci. Eng. 24 (1976) p. 247.

[19] F. Louchet, L. Kubin and D. Vesely, Philos. Mag. A 39 (1979) p. 433.

[20] S. Takeuchi and K. Maeda, Acta Metall. 25 (1977) p. 1485.

[21] D. Vesely, in Understanding Materials: A Festschrift for Sir Peter Hirsch, edited by C. J.

Humphreys, vol. (Maney Publishing, Leeds, England, 2002) p. 157.

[22] W. Sigle, Philos. Mag. A 79 (1999) p. 1009.

[23] B.G. Mendis, Y. Mishin, C.S. Hartley and K.J. Hemker, Philos. Mag. 86 (2006) p. 4607.

[24] P.D. Nellist, E.C. Cosgriff, P.B. Hirsch and D.J.H. Cockayne, Philos. Mag. 88 (2008) p. 135.

[25] J.D. Eshelby and A.N. Stroh, Philos. Mag. 42 (1951) p. 1401.

[26] M.W. Finnis and J.E. Sinclair, Philos. Mag. A 50 (1984) p. 45. 
Philosophical Magazine \& Philosophical Magazine Letters

Page 13 of 32

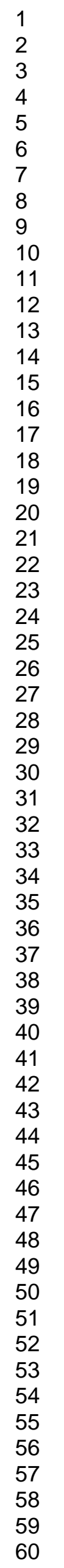

[27] M. Mrovec, D. Nguyen-Manh, D.G. Pettifor and V. Vitek, Phys. Rev. B 69 (2004) p. 094115.

[28] R. Gröger, A.G. Bailey and V. Vitek, Acta Mater. 56 (2008) p. 5401.

[29] V. Vitek, Philos. Mag. 84 (2004) p. 415.

[30] R. Gröger and V. Vitek, Philos. Mag. 89 (2009) p. 3163.

[31] J.F. Nye, Acta Metall. 1 (1953) p. 153.

[32] E. Kröner, Continuum theory of dislocations and self-stresses, (Springer-Verlag, 1958).

[33] C.T. Koch, QSTEM, http://www.christophtkoch.com/stem/index.html.

[34] C.T. Koch, Determination of core structure periodicity and point defect density along dislocations, Arizona State University, (2002).

[35] M.J. Hytch, J.-L. Putaux and J.-M. Penisson, Nature 423 (2003) p. 270.

[36] P.B. Hirsch, D.J.H. Cockayne, J. Spence and M. Whelan, Philos. Mag. 86 (2006) p. 4519.

http://mc.manuscriptcentral.com/pm-pml 


\section{Figure captions}

Figure 1: Map of screw differential displacements of the 1/2[111] screw dislocation for three slices taken from the block representing the foil at: (a) $z=+h / 2$ (upper surface), (b) $z=0$ (centre of the foil), and (c) $z=-h / 2$ (lower surface).

Figure 2: Map of edge differential displacements for the 1/2[111] screw dislocation calculated for three slices taken from the block representing the foil at: (a) $z=+h / 2$ (upper surface), (b) $z=0$ (centre of the foil), and (c) $z=-h / 2$ (lower surface). The displacements in (b) are scaled by a factor of 10 while those in (a) and (c) by a factor of 2.

Figure 3: The displacements of the Eshelby twist for (a) the upper and (b) the lower surface of the thin foil. The dots represent the positions of atoms in the three successive (111) planes adjacent to the two free surfaces; triangle shows the position of the dislocation centre. The calculated anisotropic Eshelby twist is shown in black; the isotropic Eshelby-Stroh field is in red. Note the opposite rotations in the two slices. The lengths of the arrows are scaled by a factor of 5 .

Figure 4: Comparison of the decay of the absolute displacements around the dislocation at the lower surface of the foil, $u / a$, with the distance from the dislocation, $r / a$. The purely tangential displacements obtained from the Eshelby-Stroh formula (2) are shown in red. The calculated displacements of atoms in the three (111) planes adjacent to the lower surface of the foil are plotted by black. The black dots above the Eshelby-Stroh displacements come from the atoms in the (111) plane that terminates the foil.

Figure 5: Plots of Nye tensor components for three slices taken from the upper $(z=+h / 2)$ and lower surfaces $(z=-h / 2)$ of the foil and from the middle of the foil $(z=0)$. The colours correspond to the density of the Burgers vectors of continuously distributed infinitesimal dislocations. The component $\alpha_{33}$ corresponds to the screw component; $\alpha_{31}$ and $\alpha_{32}$ to the two edge components parallel to the $x$ and $y$ axes, respectively. The circles represent the relaxed positions of atoms in the three consecutive (111) planes.

Figure 6: Simulated HREM images of: (a) a perfect crystal containing no dislocation; (b) the relaxed crystal containing the screw dislocation with atom positions given by the set of coordinates $\mathbf{x}_{f}^{i}$. 
Figure 7: The absolute displacements around the dislocation obtained from atomistic simulations (black) and from image analysis (red). The former is taken from the upper surface of the foil which is the exit surface in the image analysis. The lengths of arrows are enlarged five times.

Figure 8: Comparison of the edge component of the differential displacement maps obtained from atomistic simulations (a) and from image analysis (b). The lengths of arrows in both plots are enlarged twice. The intersection of the dislocation line with the plane of the figure is marked by the red triangle.

Figure 9: Comparison of the Nye tensor plots $\alpha_{31}$ and $\alpha_{32}$ obtained from (a) atomistically calculated positions of atoms in the three (111) planes adjacent to the upper surface of the foil, and (b) image simulations at the exit surface of the beam. 
Page 16 of 32

Philosophical Magazine \& Philosophical Magazine Letters

1
2
3
4
5
6
7
8
9
10
11
12
13
14
15
16
17
18
19
20
21
22
23
24
25
26
27
28
29
30
31
32
33
34
35
36
37
38
39
40
41
42
43
44
45
46
47
48
49
50
51
52
53
54
55
56
57
58
59
60

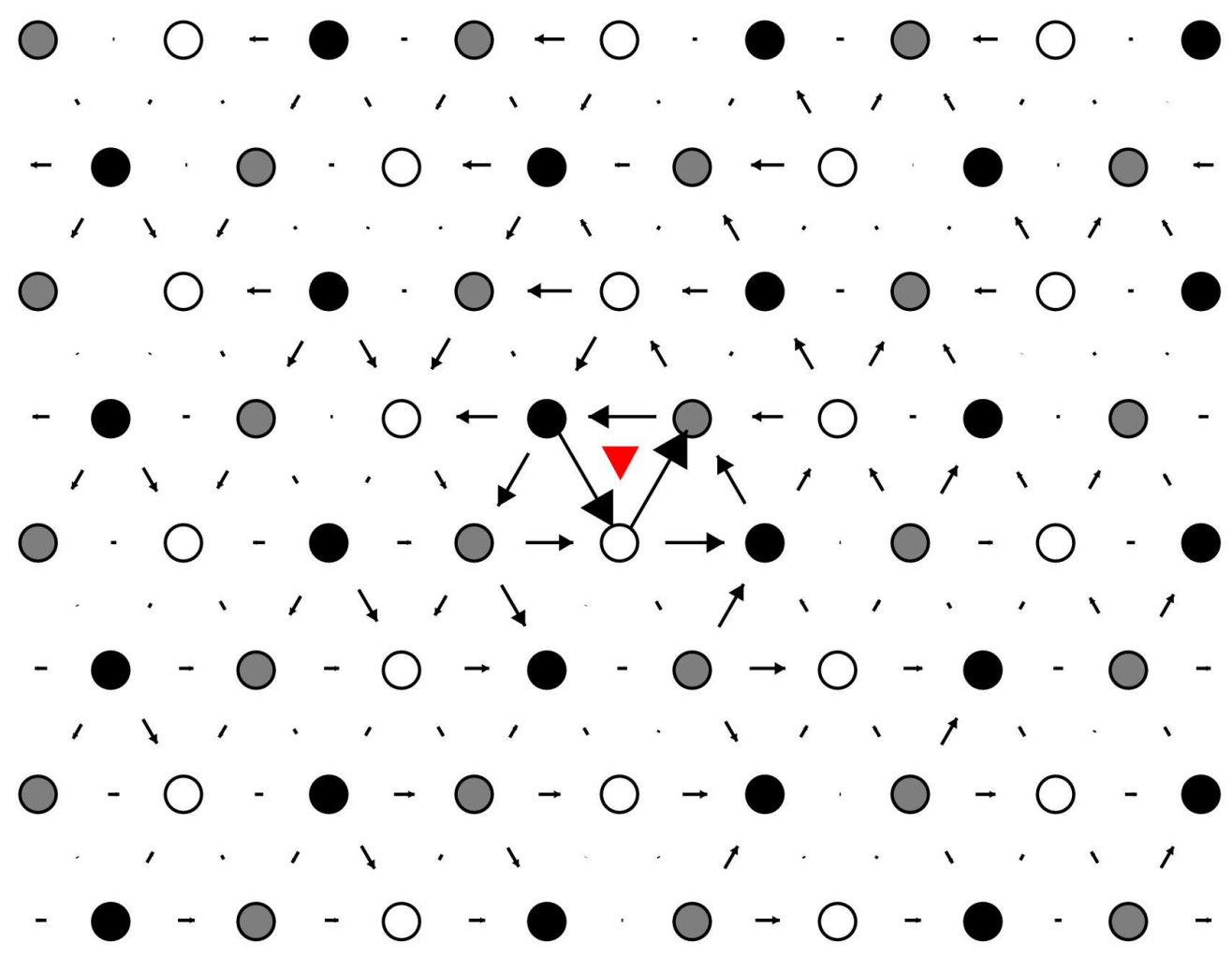

Fig. 1 (a)

$591 \times 457 \mathrm{~mm}(72 \times 72$ DPI $)$

http://mc.manuscriptcentral.com/pm-pml 
Philosophical Magazine \& Philosophical Magazine Letters

Page 17 of 32

\begin{tabular}{l}
1 \\
2 \\
3 \\
4 \\
5 \\
6 \\
7 \\
8 \\
9 \\
10 \\
11 \\
12 \\
13 \\
14 \\
15 \\
16 \\
17 \\
18 \\
19 \\
20 \\
21 \\
22 \\
23 \\
24 \\
25 \\
26 \\
27 \\
28 \\
29 \\
30 \\
31 \\
32 \\
33 \\
34 \\
35 \\
36 \\
37 \\
38 \\
39 \\
40 \\
4 \\
4 \\
52 \\
50 \\
53 \\
53 \\
4 \\
45 \\
46 \\
4 \\
4 \\
4 \\
50 \\
5 \\
\hline
\end{tabular}
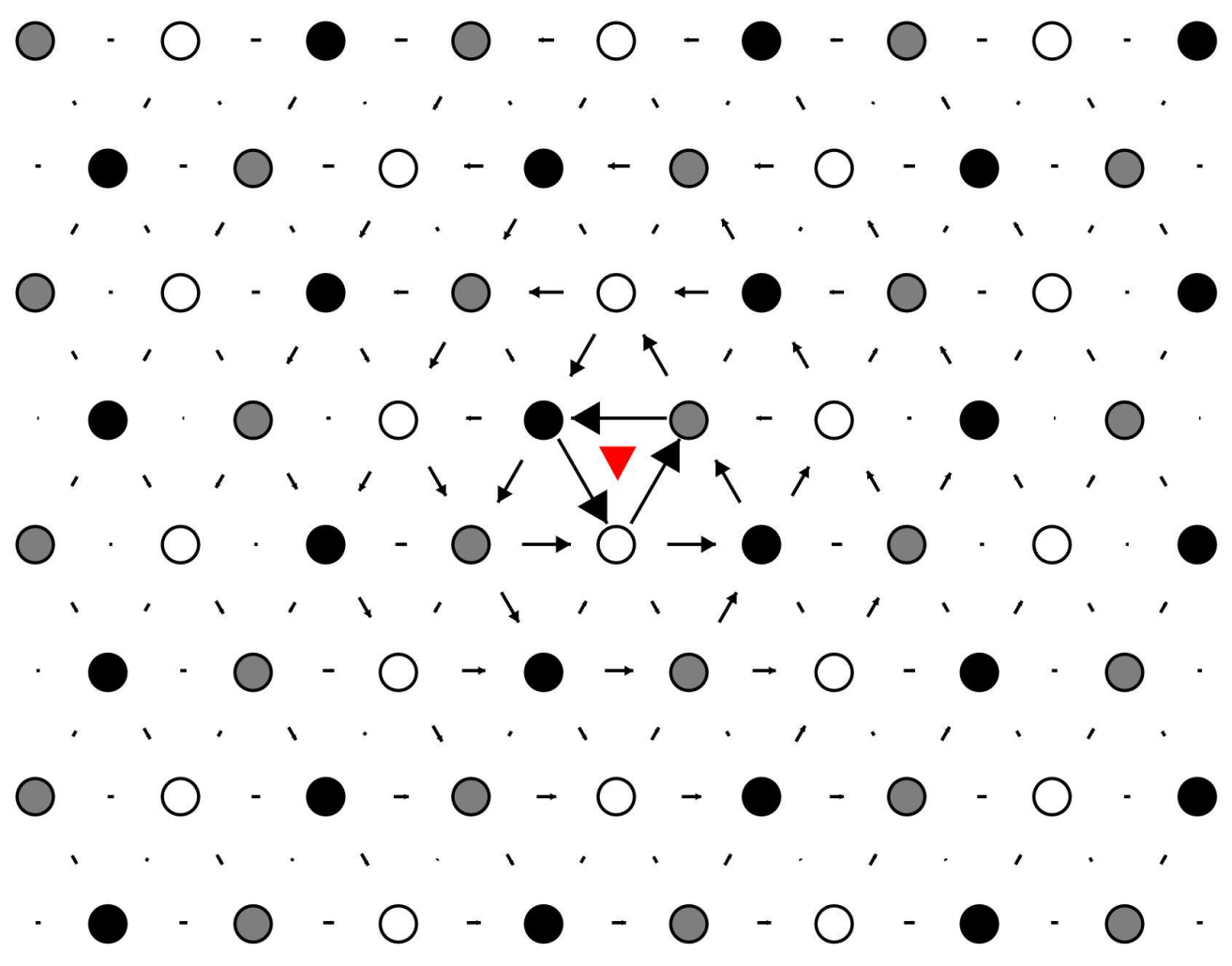

Fig. 1 (b)

$591 \times 456 \mathrm{~mm}(72 \times 72$ DPI $)$

http://mc.manuscriptcentral.com/pm-pml 
Page 18 of 32

Philosophical Magazine \& Philosophical Magazine Letters

\begin{tabular}{l}
1 \\
2 \\
3 \\
4 \\
5 \\
6 \\
7 \\
8 \\
9 \\
10 \\
11 \\
12 \\
13 \\
14 \\
15 \\
16 \\
17 \\
18 \\
19 \\
20 \\
21 \\
22 \\
23 \\
24 \\
25 \\
26 \\
27 \\
28 \\
29 \\
30 \\
31 \\
32 \\
33 \\
34 \\
35 \\
36 \\
37 \\
38 \\
39 \\
40 \\
41 \\
42 \\
43 \\
44 \\
45 \\
46 \\
47 \\
48 \\
59 \\
50 \\
51 \\
52 \\
5 \\
\hline
\end{tabular}
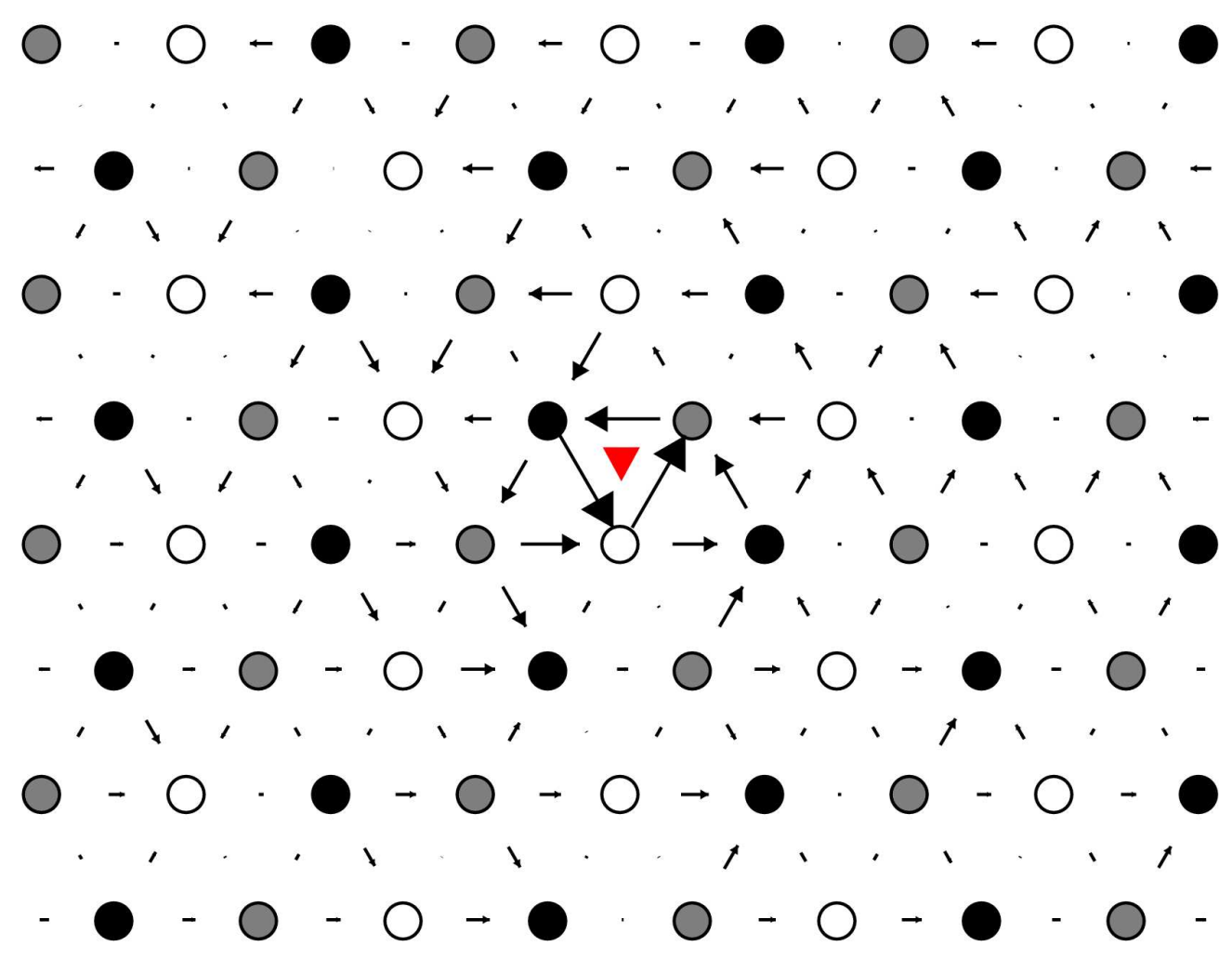

Fig. 1 (c)

$590 \times 456 \mathrm{~mm}(72 \times 72$ DPI $)$

http://mc.manuscriptcentral.com/pm-pml 


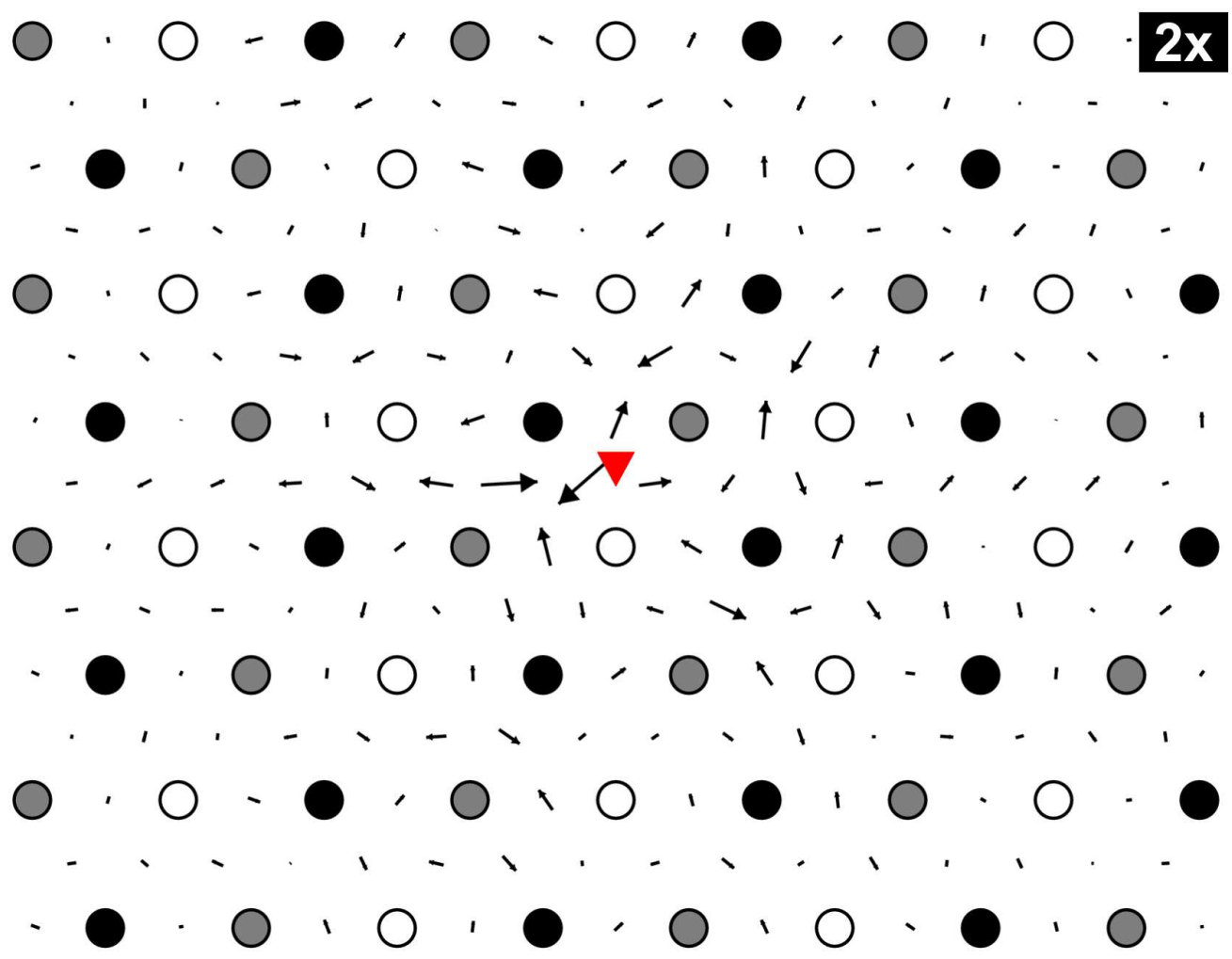

Fig. 2 (a)

$591 \times 457 \mathrm{~mm}(72 \times 72$ DPI $)$ 
Page 20 of 32

Philosophical Magazine \& Philosophical Magazine Letters

\begin{tabular}{l}
1 \\
2 \\
3 \\
4 \\
5 \\
6 \\
7 \\
8 \\
9 \\
10 \\
11 \\
12 \\
13 \\
14 \\
15 \\
16 \\
17 \\
18 \\
19 \\
20 \\
21 \\
22 \\
23 \\
24 \\
25 \\
26 \\
27 \\
28 \\
29 \\
30 \\
31 \\
32 \\
33 \\
34 \\
35 \\
36 \\
37 \\
38 \\
39 \\
40 \\
41 \\
42 \\
43 \\
44 \\
45 \\
46 \\
47 \\
48 \\
59 \\
50 \\
51 \\
52 \\
5 \\
\hline
\end{tabular}

$0 \cdot 0 \cdot \bullet \cdot 0 \cdot 0 \cdot \bullet \cdot 0 \cdot 0$ 10x
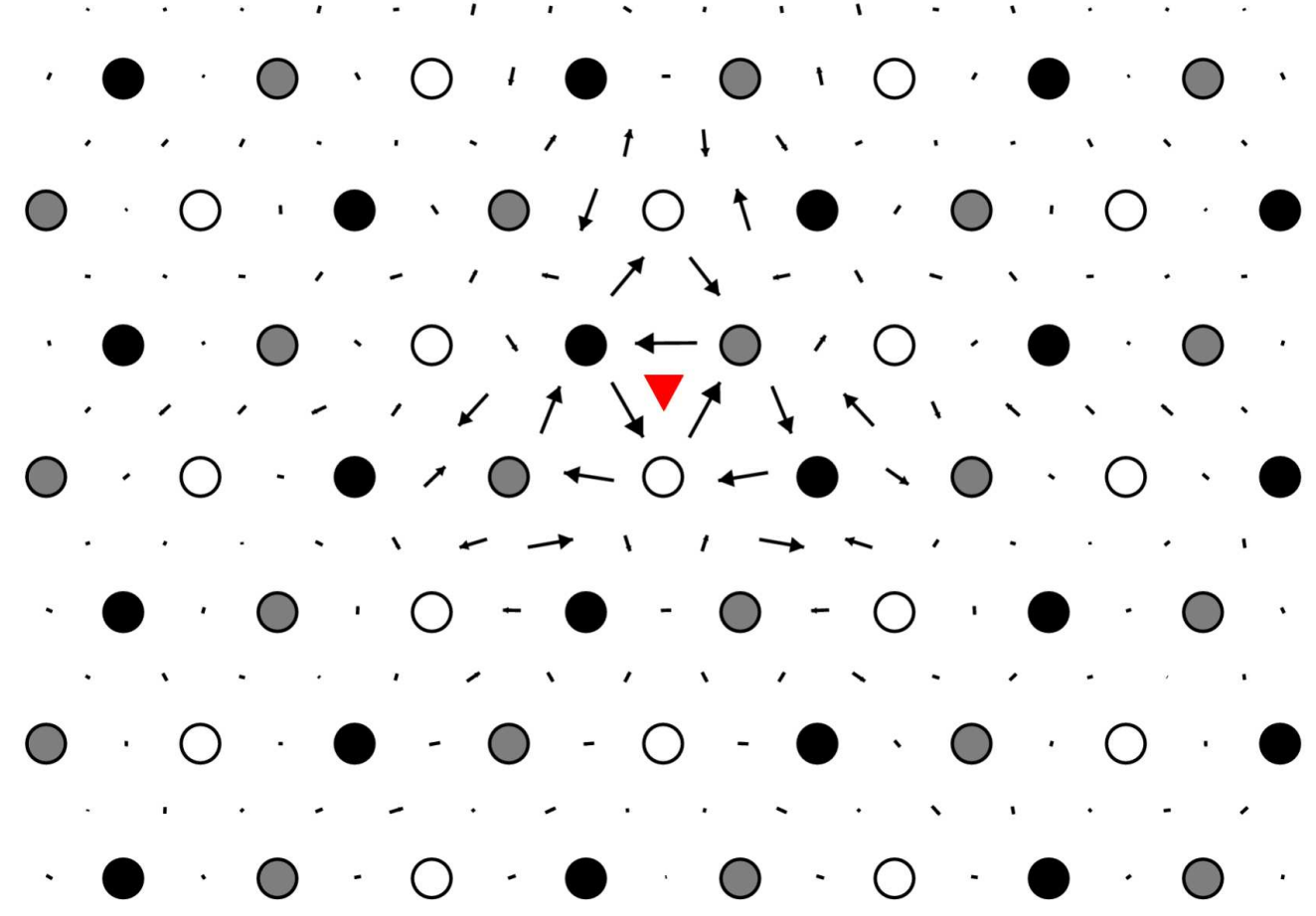

Fig. 2 (b)

$591 \times 456 \mathrm{~mm}(72 \times 72$ DPI $)$

http://mc.manuscriptcentral.com/pm-pml 


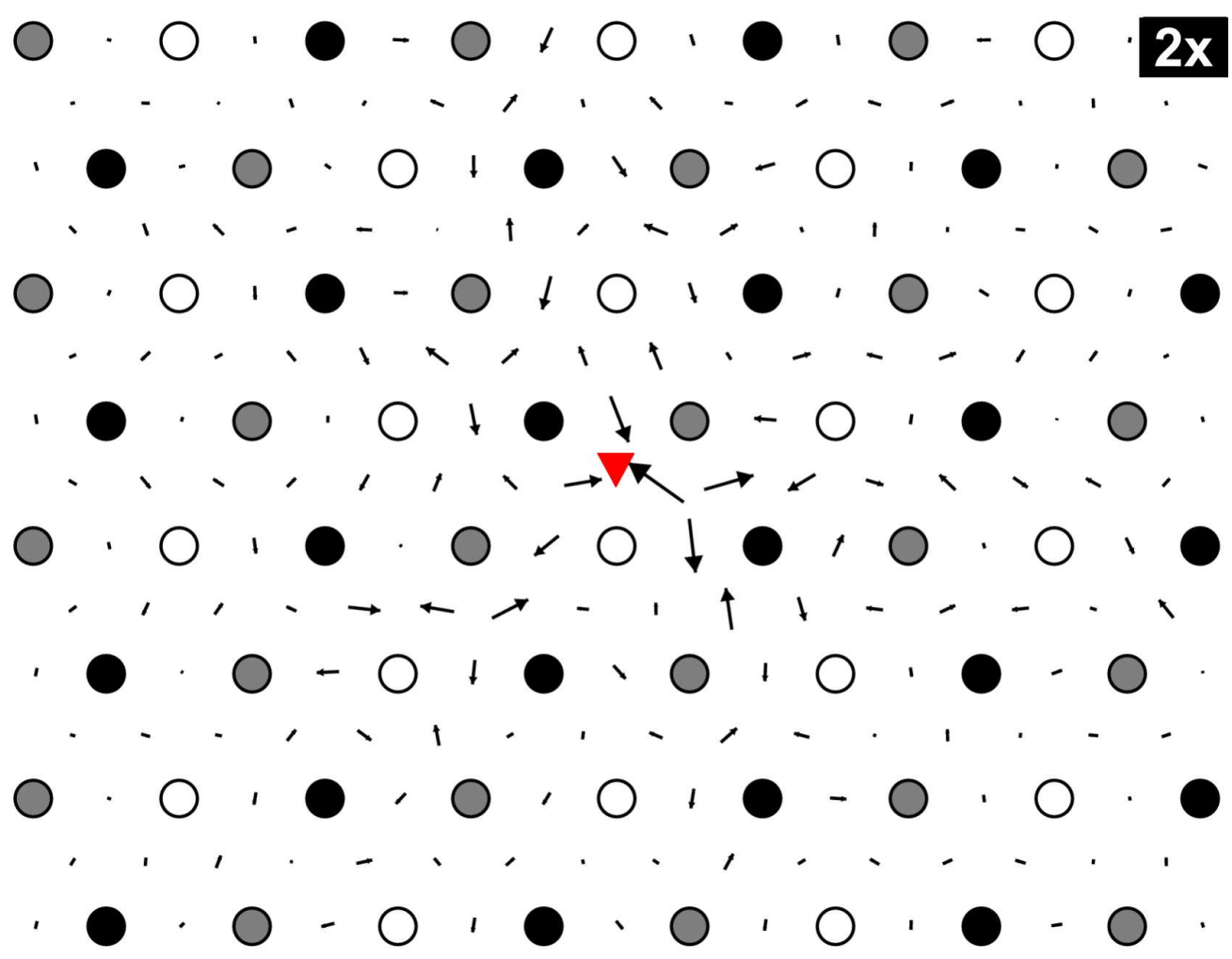

Fig. 2 (c)

$591 \times 456 \mathrm{~mm}(72 \times 72$ DPI $)$ 


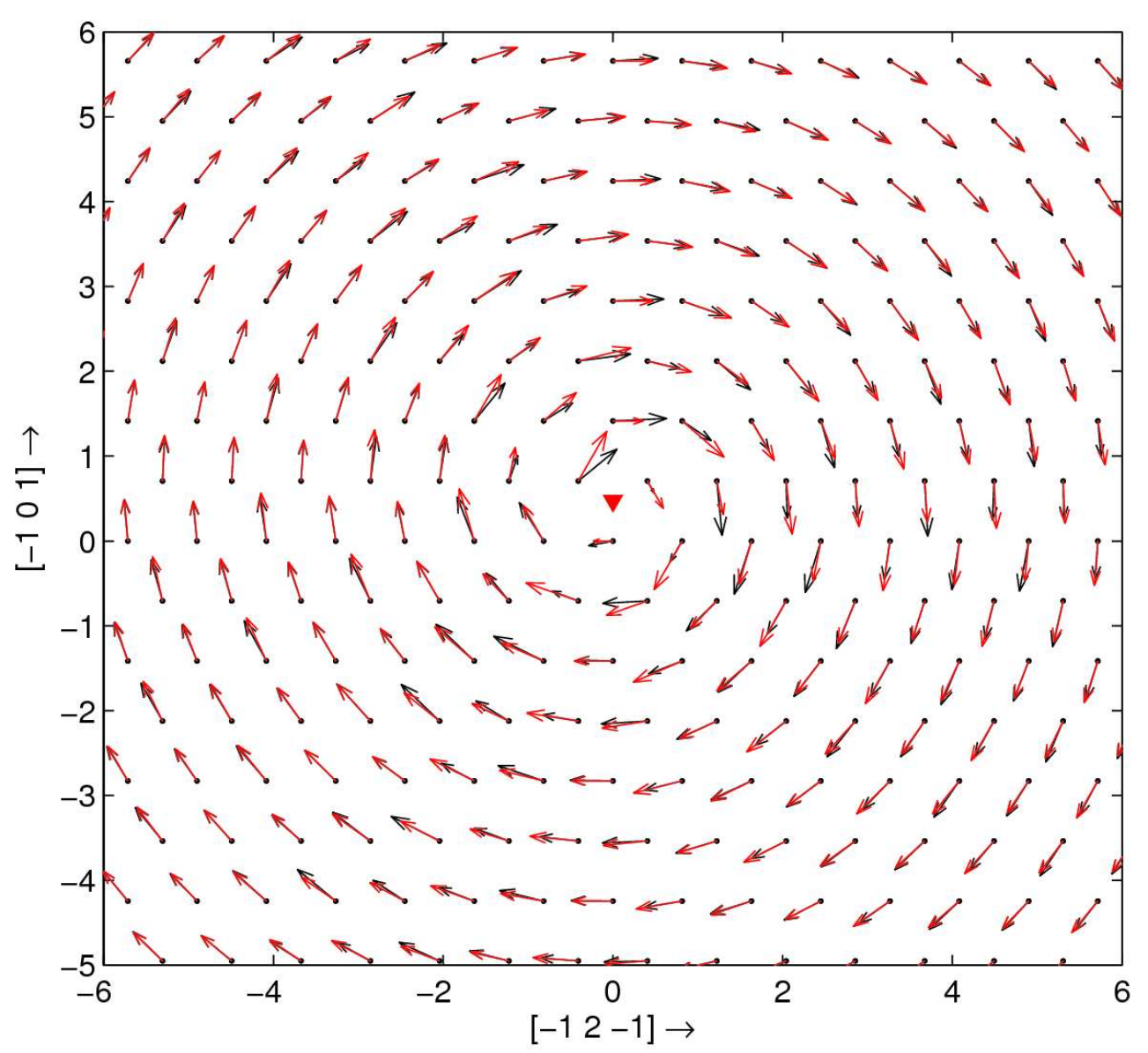

Fig. 3 (a)

$152 \times 139 \mathrm{~mm}(200 \times 200$ DPI $)$

http://mc.manuscriptcentral.com/pm-pml 


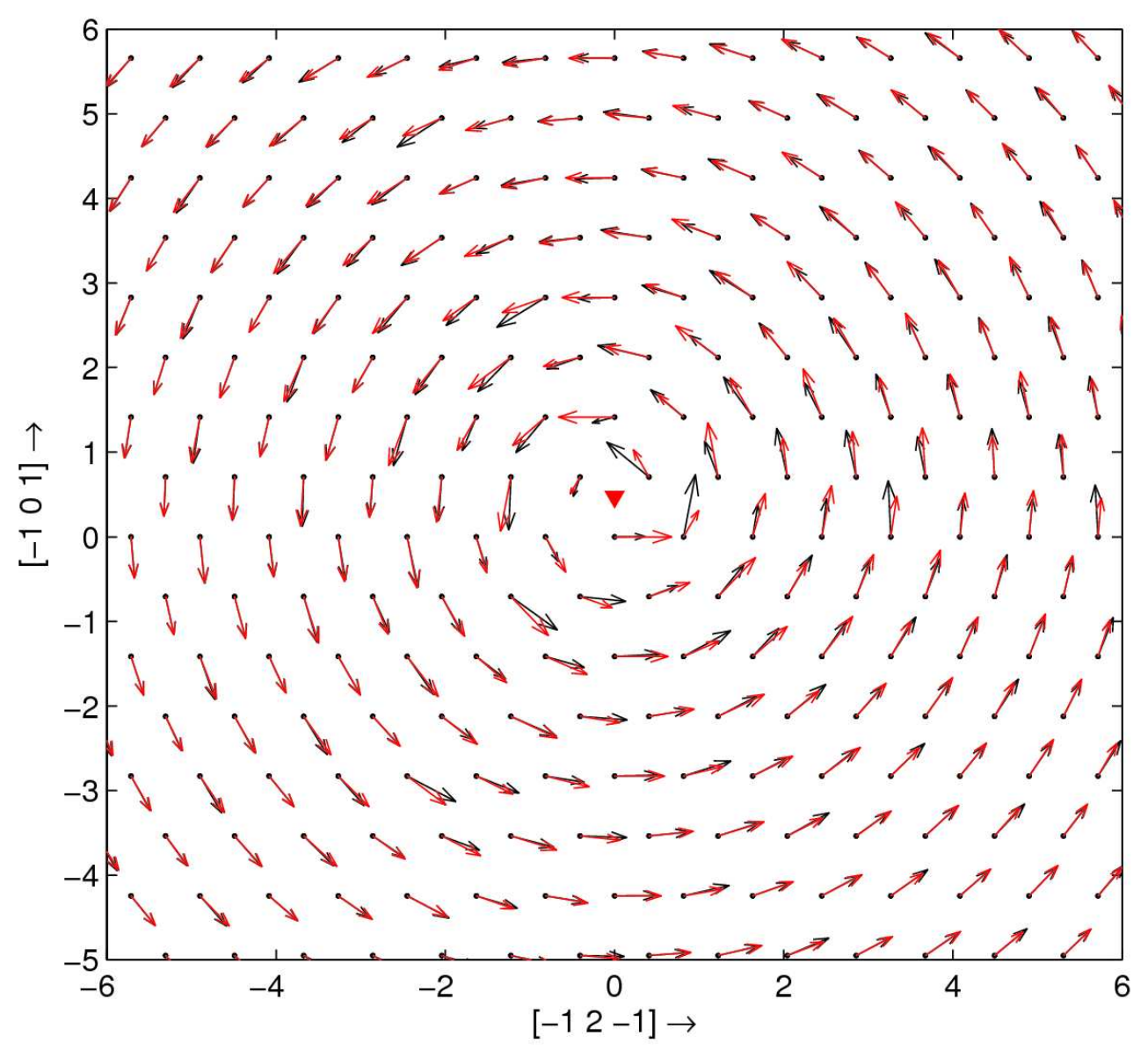

Fig. 3 (b)

$152 \times 139 \mathrm{~mm}(200 \times 200$ DPI $)$

http://mc.manuscriptcentral.com/pm-pml 


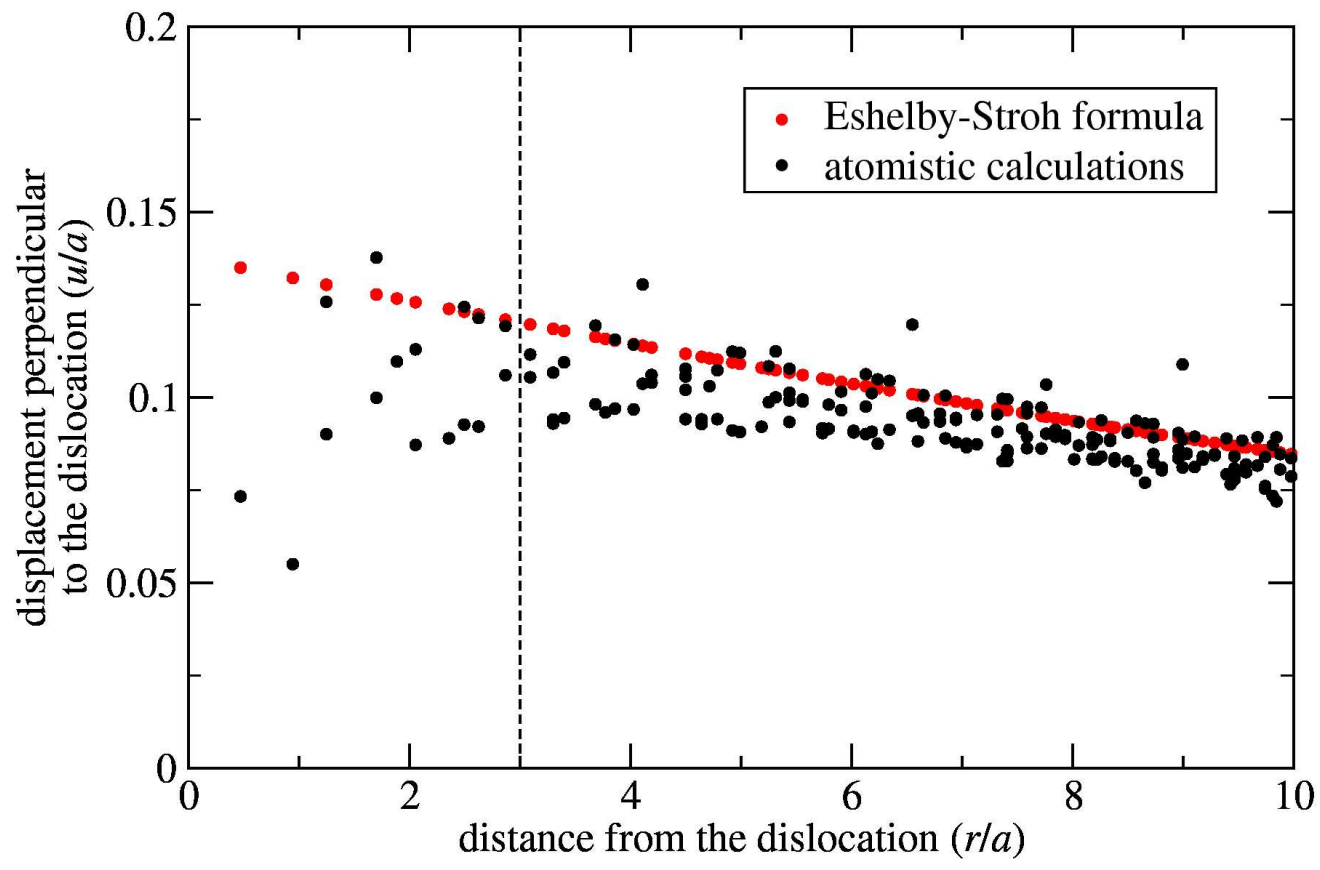

Fig. 4

$227 \times 148 \mathrm{~mm}(200 \times 200$ DPI $)$ 


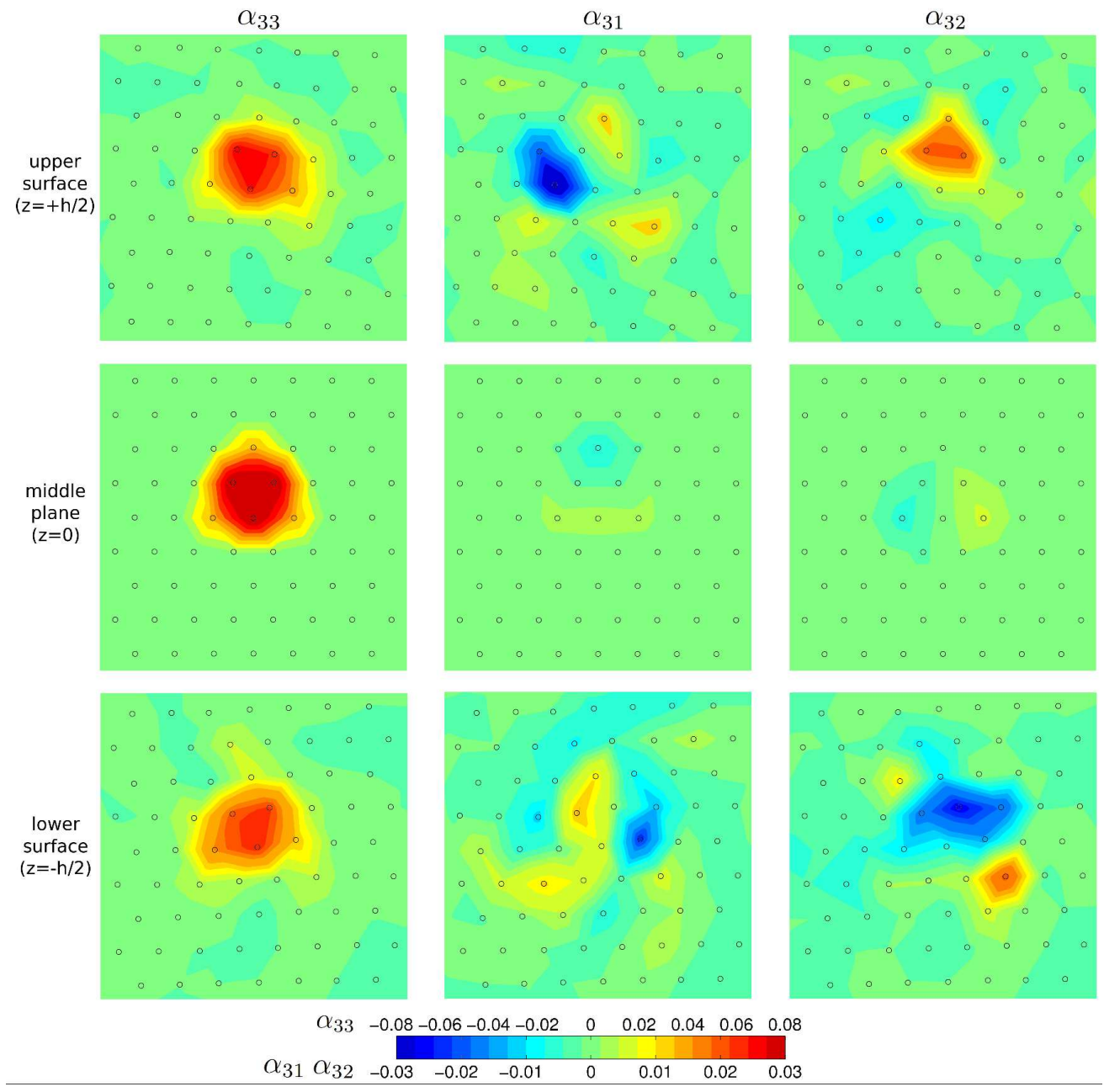

Fig. 5

$1235 \times 1235 \mathrm{~mm}(72 \times 72 \mathrm{DPI})$ 


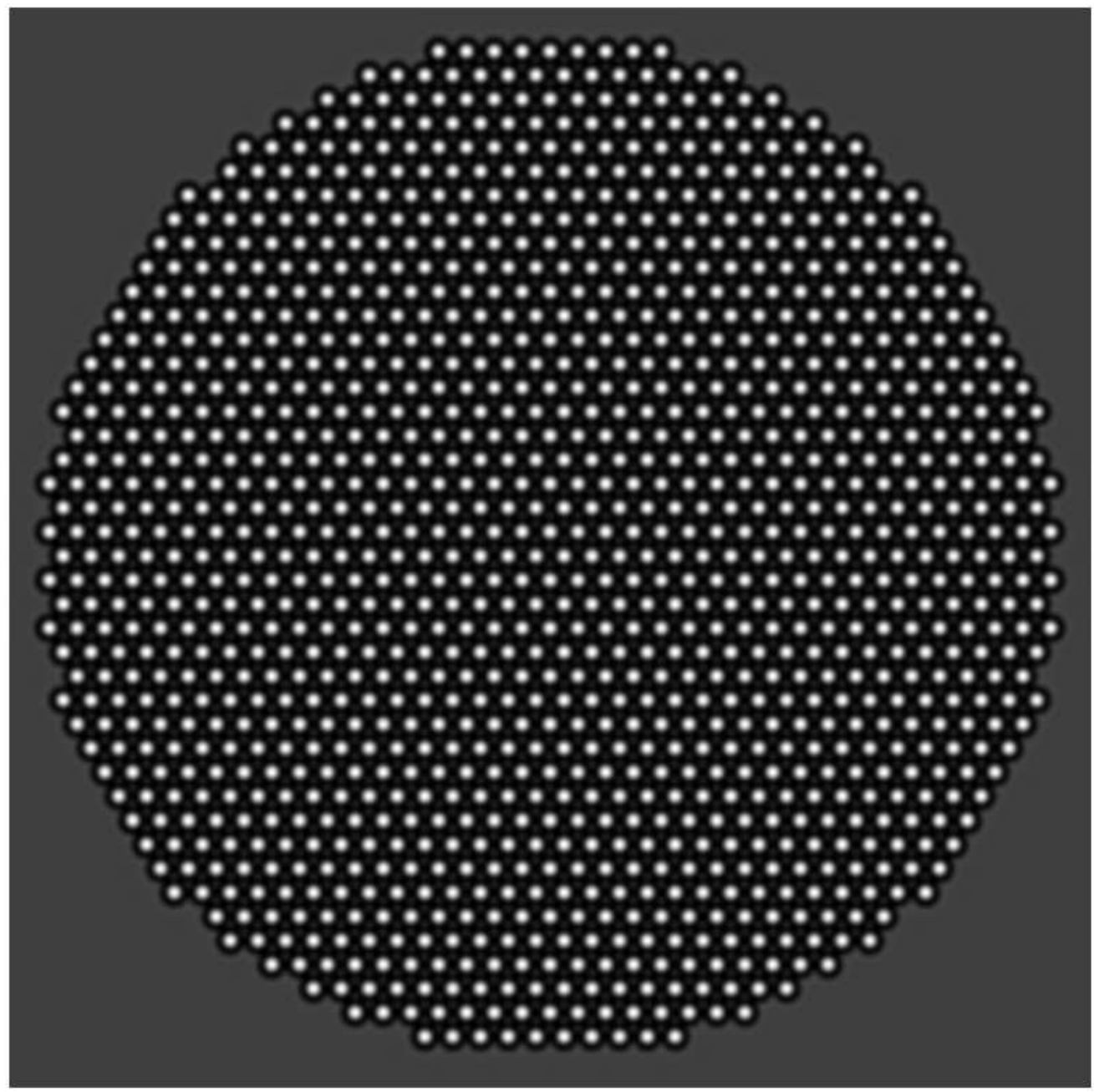

Fig. 6 (a) 


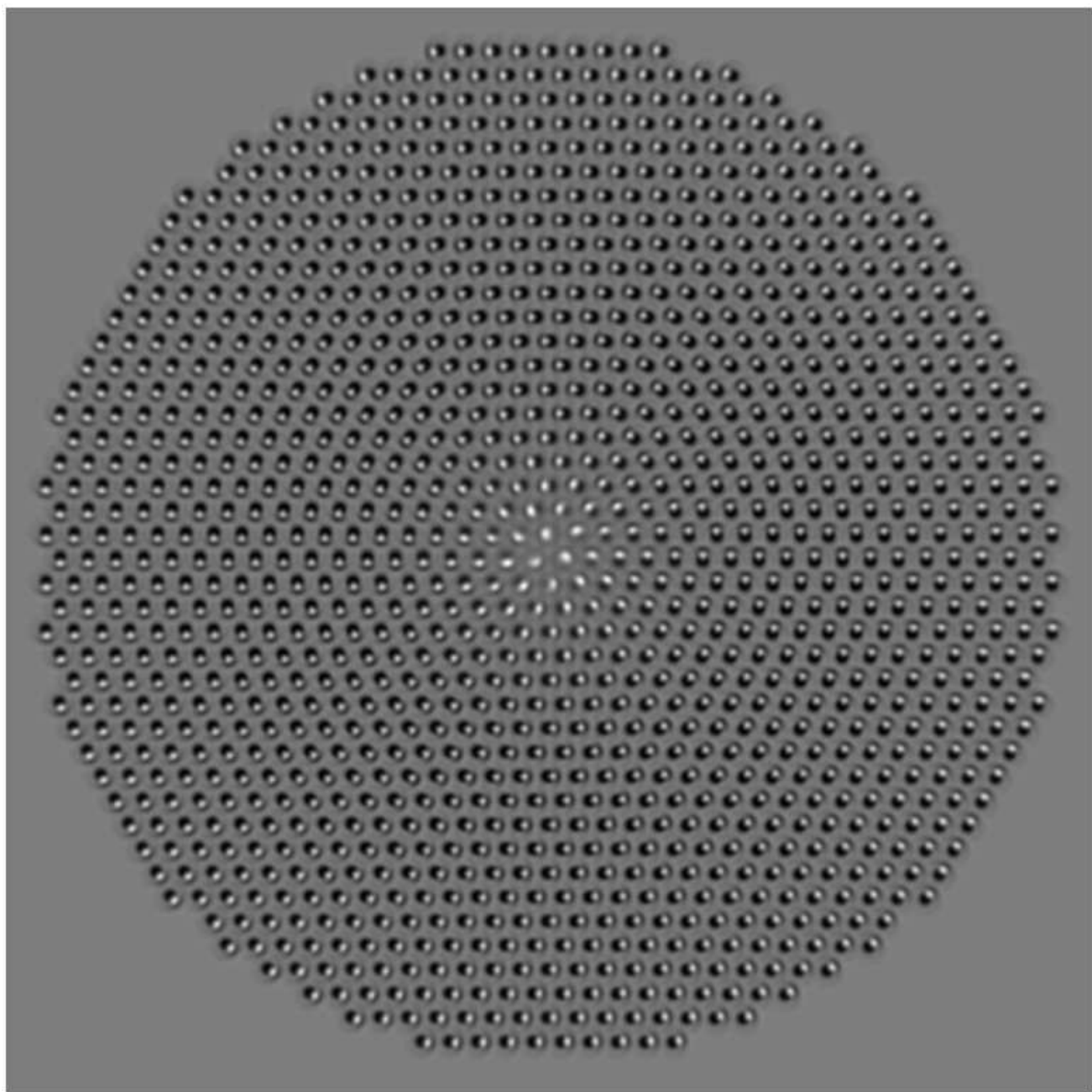

Fig. 6 (b) 


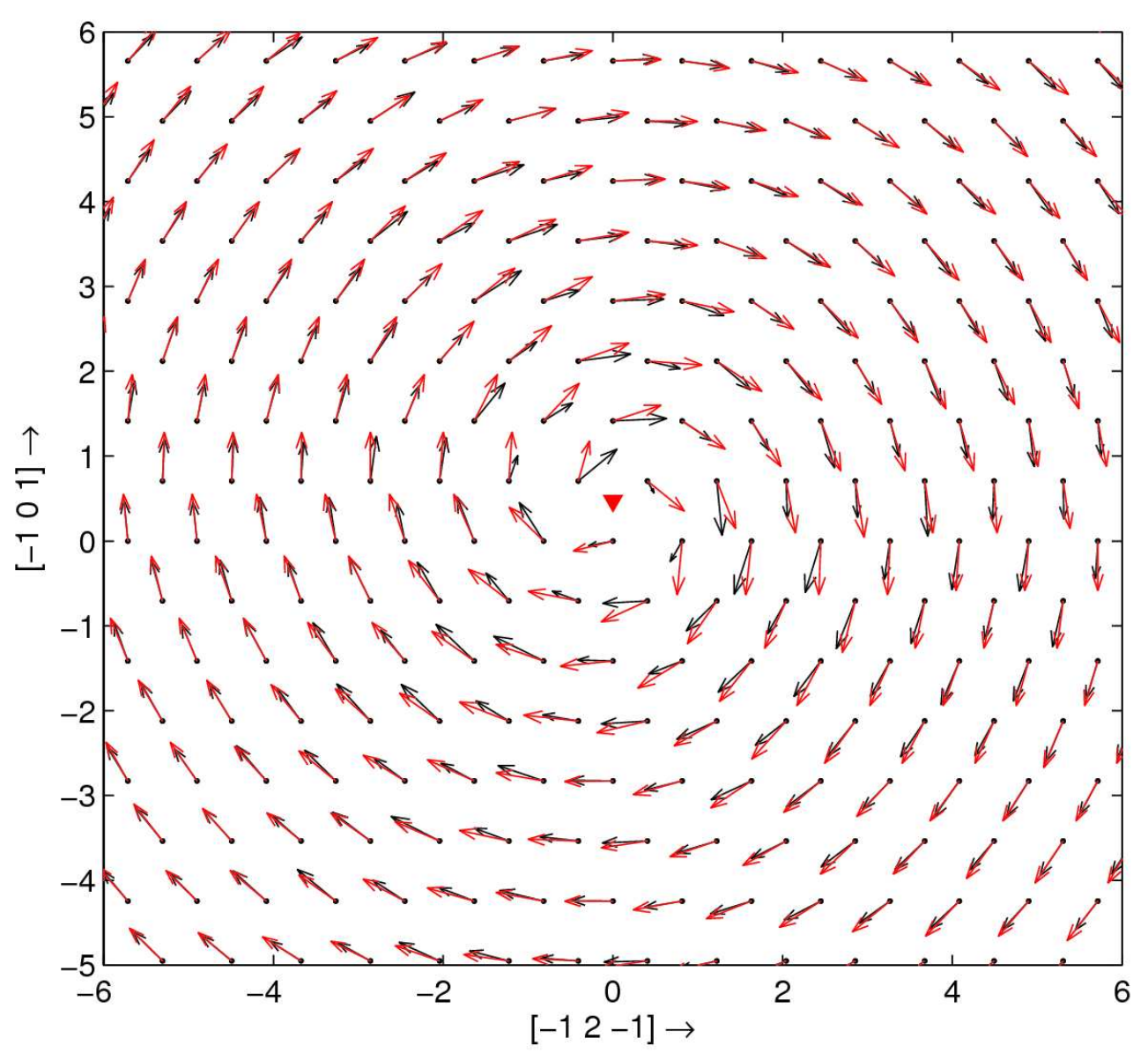

Fig. 7

$152 \times 139 m m(200 \times 200$ DPI $)$ 
Philosophical Magazine \& Philosophical Magazine Letters

Page 29 of 32

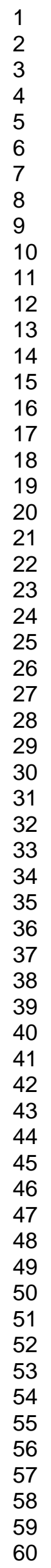

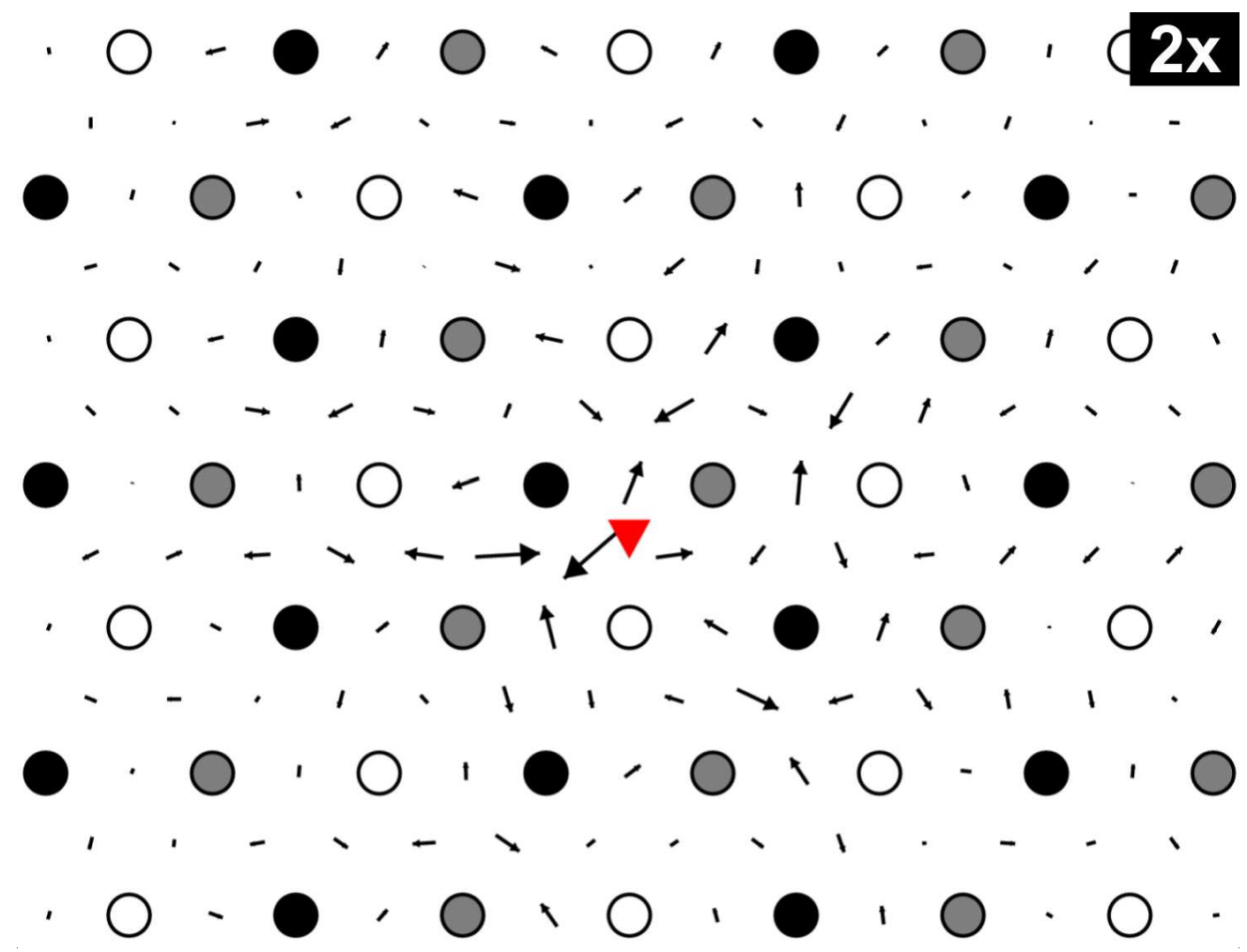

Fig. 8 (a)

$516 \times 395 \mathrm{~mm}(72 \times 72$ DPI $)$

http://mc.manuscriptcentral.com/pm-pml 


1
2
3
4
5
6
7
8
9
10
11
12
13
14
15
16
17
18
19
20
21
22
23
24
25
26
27
28
29
30
31
32
33
34
35
36
37
38
39
40
41
42
43
44
45
46
47
48
49
50
51
52
53
54
55
56
57
58
60

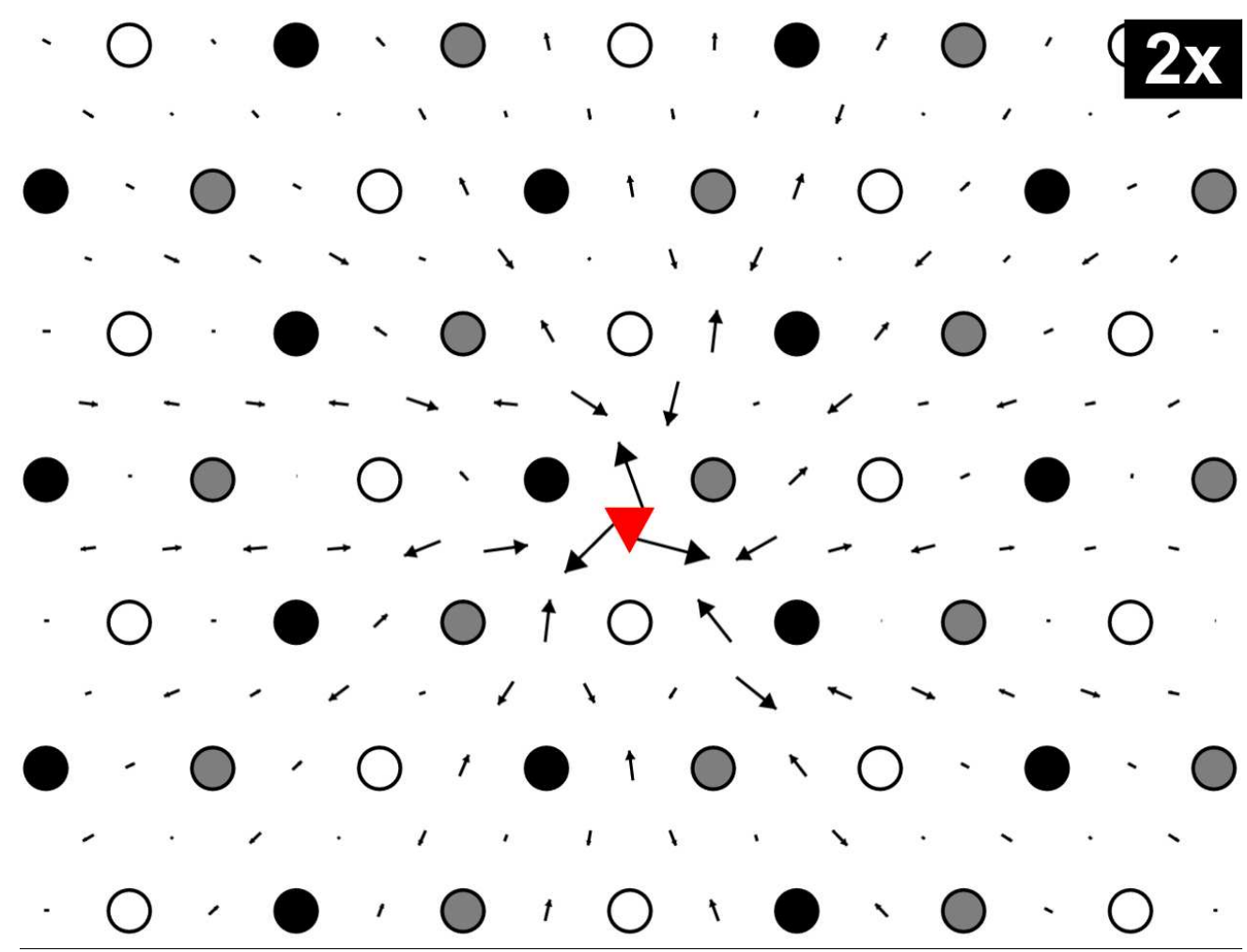

Fig. 8 (b)

$446 \times 339 \mathrm{~mm}(72 \times 72 \mathrm{DPI})$ 


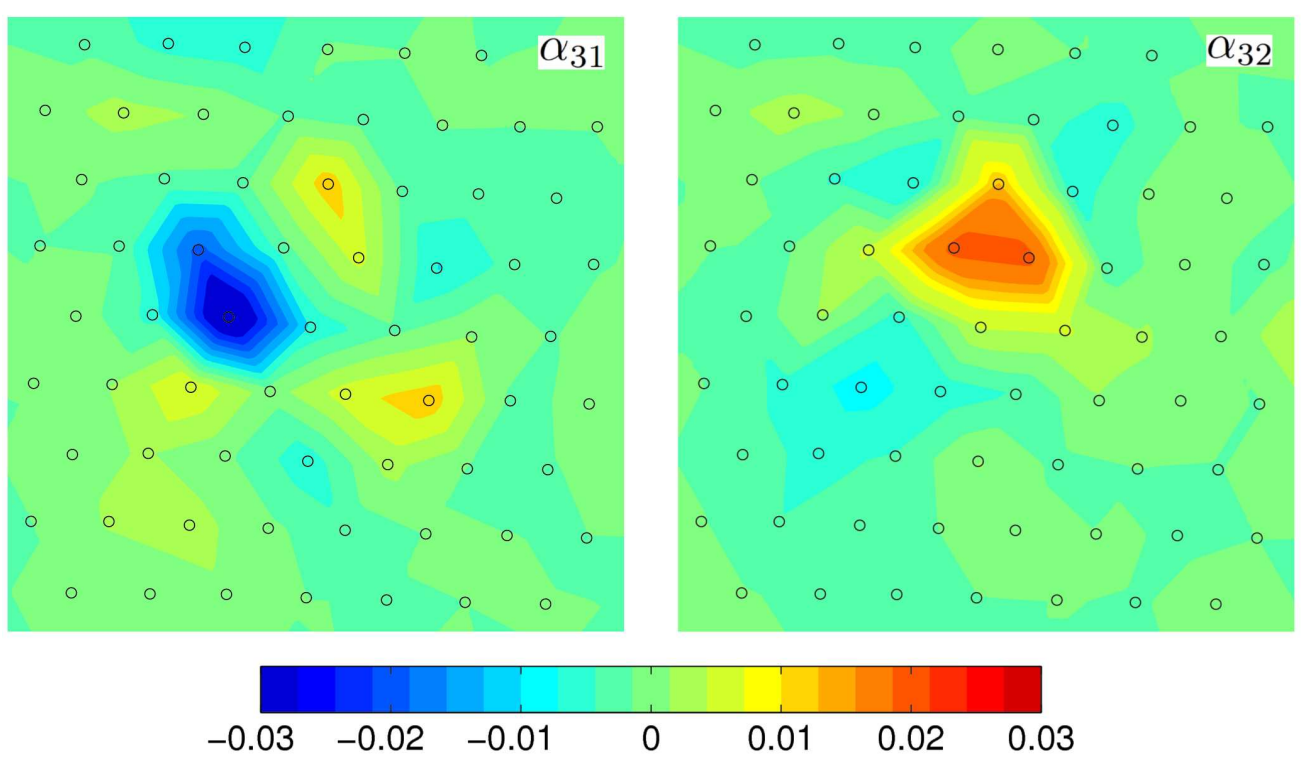

Fig. 9 (a)

$731 \times 423 \mathrm{~mm}(72 \times 72 \mathrm{DPI})$

http://mc.manuscriptcentral.com/pm-pml 

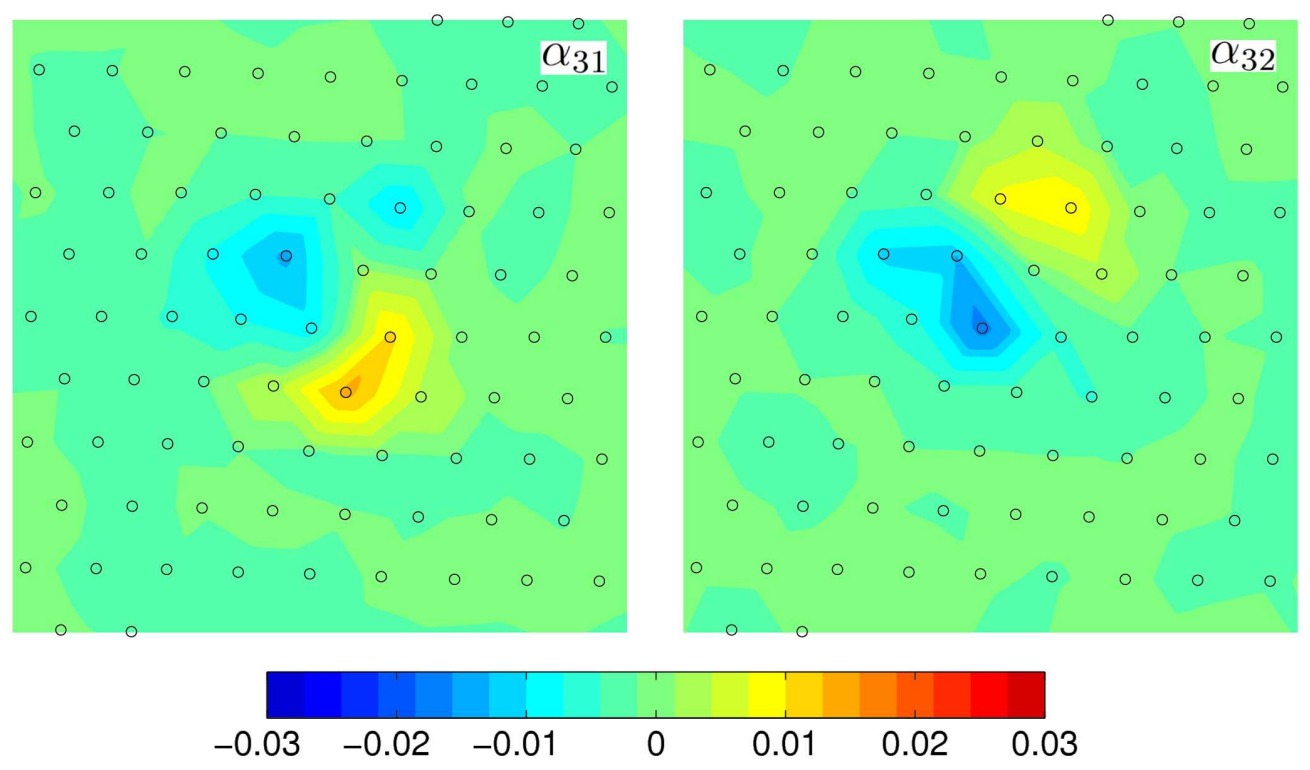

Fig. 9 (b)

$732 \times 426 \mathrm{~mm}(72 \times 72$ DPI $)$ 